Discussion Paper No. 971

\author{
STRATEGY-PROOFNESS \\ AND EFFICIENCY \\ FOR NON-QUASI-LINEAR \\ COMMON-TIERED-OBJECT \\ PREFERENCES: \\ CHARACTERIZATION \\ OF MINIMUM PRICE RULE
}

Yu Zhou

Shigehiro Serizawa

May 2016

The Institute of Social and Economic Research

Osaka University

6-1 Mihogaoka, Ibaraki, Osaka 567-0047, Japan 


\title{
Strategy-Proofness and Efficiency for Non-quasi-linear Common-Tiered-Object Preferences: \\ Characterization of Minimum Price Rule*
}

\author{
$\mathrm{Yu} \mathrm{Zhou}^{\dagger} \quad$ Shigehiro Serizawa ${ }^{\ddagger}$
}

May 27, 2016

\begin{abstract}
We consider the allocation problem of assigning heterogenous objects to a group of agents and determining how much they should pay. Each agent receives at most one object. Agents have non-quasi-linear preferences over bundles, each consisting of an object and a payment. Especially, we focus on the cases: (i) objects are linearly ranked, and as long as objects are equally priced, agents commonly prefer a higher ranked object to a lower ranked one, and (ii) objects are partitioned into several tiers, and as long as objects are equally priced, agents commonly prefer an object in the higher tier to an object in the lower tier. The minimum price rule assigns a minimum price (Walrasian) equilibrium to each preference profile. We establish: (i) on a common-object-ranking domain, the minimum price rule is the only rule satisfying efficiency, strategy-proofness, individual rationality and no subsidy, and (ii) on a common-tiered-object domain, the minimum price rule is the only rule satisfying these four axioms.
\end{abstract}

Keywords: strategy-proofness, efficiency, non-quasi-linearity, minimum price rule, common-object-ranking domain, common-tiered-object domain

JEL Classification: D44, D61, D71, D82

*The preliminary version of this article was presented at the12th Meeting of the Society for Social Choice and Welfare, Academia Sinica-ISER Economics Workshop 2014, 2014 SSK International Conference on Distributive Justice in Honor of Professor William Thomson, 2014 ISER Market Design Workshop, ISI-ISER Young Economists Workshop 2015, and the Conference on Economic Design 2015. We thank participants at those conferences and workshops for their comments. We also thank Kazuhiko Hashimoto, Tomoya Kazumura, Takehito Masuda, Debasis Mishra, Shuhei Morimoto, James Schummer, Arunava Sen, Ning Sun, Jingyi Xue, Ryan Tierney, and Huaxia Zeng for their helpful comments. Especially, we are grateful to William Thomson for his detailed discussion. We gratefully acknowledge financial support from the Joint Usage/Research Center at ISER, Osaka University, and the Japan Society for the Promotion of Science (15J01287, 15H03328, and 15H05728).

${ }^{\dagger}$ Graduate School of Economics, Osaka University 1-7, Machikaneyama, Toyonaka, Osaka 560-0043, Japan. Email: zhouyu_0105@hotmail.com

¥Institute of Social and Economic Research, Osaka University, 6-1, Mihogaoka, Ibaraki, Osaka 567-0047, Japan. Email: serizawa@iser.osaka-u.ac.jp 


\section{Introduction}

We consider the allocation problem of assigning heterogenous objects to a group of agents and determining how much each agent should pay. Each agent receives at most one object. Agents have non-quasi-linear preferences over bundles, each consisting of an object and a payment. Non-quasi-linear preferences describe the environment where changing the same amount of money at different payments for a given object exerts different impacts on the benefit deriving from consuming that bundle. In addition to the non-quasi-linearity, the allocation problem we investigate also has the following features, which are exemplified below:

Example A: Central business districts are located in the city center where households are employed and commute everyday with same public transportation system. Houses are similar in qualities and sizes, but much different in the distances from the city center. Each household needs at most one house. As long as houses are equally priced, households prefer a house with shorter distance to the city center to the one with longer distance, since longer distance takes more commuting fee and time. However, when several houses have the same distance to the city center, even if those houses are equally prices, households might have different preferences on them. Since the purchase of houses has a great impact on the budget of most households, each household has non-quasi-linear preferences over houses and payments. ${ }^{1}$

Example B: Several condominiums belong to the same building and similar in qualities and sizes. Each household needs at most one condominium. As long as condominiums are equally priced, households commonly prefer condominiums in higher floors to those in lower floors. However, households might have different preferences on condominiums on the same floor even if they are equally prices. Similarly to Example 1, each household has non-quasi-linear preferences over condominiums and payments.

The above examples introruce our special focus of the non-quasi-linear environment:

(i) Objects are linearly ranked, and as long as objects are equally priced, agents commonly prefer a higher ranked object to a lower ranked one.

(ii) Objects are partitioned into several tiers, and as long as objects are equally priced, agents commonly prefer an object in the higher tier to an object in the lower tier. However, even if objects are equally priced, agents may have different preferences over the objects in the same tier.

We try to identify the (allocation) rules satisfying efficiency, strategy-proofness, individual rationality and no subsidy for above-mentioned allocation problems defined on the commonobject-ranking domain and common-tiered-object domain. An allocation specifies how the objects are allocated and how each agent should pay. A rule is a mapping from the set of agents' preference profiles (called "domain") to the set of allocations. The common-object-ranking domain contains a set of preference profiles where for each preference profile, individual preference satisfies money monotonicity, object monotonicity, possibility of compensation, and more importantly, commonly ranks objects according to some object permutation. The commontiered-object domain contains a set of preference profiles where for each preference profile, in

\footnotetext{
${ }^{1}$ The housing market in a monocentricity has been investigated under different contents by Kaneko (1983), Kaneko, et al (2006) and Sai (2016) etc.
} 
addition to the previous first four assumptions, objects are partitioned into several tiers and individual preference commonly ranks objects according to the tier partition. An allocation is efficient if no one can be better off without reducing others' welfare or reducing the total amount of the payments. Efficiency describes the property of a rule that for each preference profile, the rule always selects the efficient allocation. Strategy-proofness says that for each agent and each preference profile, truthfully revealing the private information is always a weakly dominant strategy. Individual rationality says that for each agent and each preference profile, everyone should be no worse than getting and paying nothing. This property guarantees the agents' voluntary participations. For the last property, no subsidy, it just says that the payment for each object is non-negative.

The "minimum price (Walrasian) rule" is an important rule satisfying the above-mentioned four properties. In our model, the set of equilibrium prices forms a non-empty complete lattice and the minimum (Walrasian) equilibrium price vector is well defined. ${ }^{2}$ The minimum price rule is a rule that given each preference profile, it always selects an equilibrium with the minimum price vector. We establish: (i) on a common-object-ranking domain, a rule satisfies efficiency, strategy-proofness, individual rationality and no subsidy if and only if it is the minimum price rule; and (ii) on a common-tiered-object domain, the minimum price rule is the only rule satisfying these four axioms.

Efficient and strategy-proof rules for non-quasi-linear preferences have already been studied by the literature. ${ }^{3}$ Much attention has been paid to the model where the set of equilibrium prices or payoffs has the lattice structure. In the one-to-one two-sided matching model with money transfer, although the set of equilibrium payoffs forms a non-empty complete lattice, no rule satisfies efficiency and strategy-proofness, in addition to individual rationality and no pairwise budget deficit. However, if strategy-proofness is weakened to one-sided strategy-proofness, the one-sided optimal core rule satisfies this property in addition to efficiency, individual rationality and no pairwise budget deficit. Furthermore, it is the only rule satisfying those properties (Demange and Gale, 1985; Morimoto, 2016).

As a special case of the one-to-one two-sided matching model with money transfer, object assignment models with money transfer have also been studied. ${ }^{4}$ In these models, the set of equilibrium prices also forms a non-empty complete lattice and consequently, the minimum price (the agent-sided optimal core allocation) rule is well defined. Two strands of literature address this issue.

One strand analyzes the case where objects are identical. In this case, the minimum price rule is equivalent to the Vickrey rule (Vickrey, 1961). The Vickrey rule is the only rule satisfying efficiency and strategy-proofness, in addition to individual rationality and no subsidy (Saitoh and Serizawa, 2008; Sakai, 2008). Moreover, the Vickrey rule is the only rule satisfying those

\footnotetext{
${ }^{2}$ See Fact 1 and Fact 2 for details.

${ }^{3}$ Some authors also investigate the strategy-proof and fair rules for the non-quasi-linear preferences, for example, Alkan et al, (1991), Sun and Yang (2003), Andersson, et al, (2010), Adachi (2014), and Tierney (2015) etc. Recently, Baisa (2015a, 2015b) investigates the auction models for the non-quasi-linear preferences.

${ }^{4}$ Assuming each agent at most receives one object is also important for identifying efficient and strategy-proof rules for non-quasi-linear preferences. Recently, Kazumura and Serizawa (2015) relax this assumption and show some impossibility results.
} 
properties on subdomains including preferences only exhibiting positive or negative income effects.

The other strand analyzes the case where objects are heterogenous. In this case, the Vickrey rule is not equivalent to the minimum price rule. ${ }^{5}$ The minimum price rule is the only rule satisfying efficiency, strategy-proofness, individual rationality and no subsidy for losers (Morimoto and Serizawa, 2015). Housing markets with bounded house prices and existing tenants are also studied recently. In those models, the minimum price rule may not be well defined. However, with some mild conditions, there still are some (constraint) efficient and strategy-proof rules (Andersson and Svensson, 2014; Andersson et al, 2016).

This paper is a further study of the efficient and strategy-proof rules on the restricted non-quasi-linear domains for the heterogenous objects case. Although Morimoto and Serizawa (2015) already establish the characterization on a larger domain by using similar axioms, our results are independent of them in the following points.

First, our focus are the smaller domains, common-object-ranking domains and commontiered-object domains. The above properties of rules are weaker on those domains than on the domain of Morimoto and Serizawa (2015). When we analyze the allocation problems exemplified above, the domain of Morimoto and Serizawa (2015) includes unsuitable preferences and their results cannot be applied.

Second, although we owe some proof structure to Morimoto and Serizawa (2015) to establish the characterizations, the detailed contents of the proofs are different. In addition, most of our proofs do not impose any restrictions on the numbers of agents and objects while the assumption that the number of agents is larger than the number of objects plays an important role in the proof of Morimoto and Serizawa (2015)'s characterization.

The common-tiered-object domain has already been studied to identify the efficient and strategy-proof rules in the two-sided matching model without money transfer and probabilistic assignment model without money transfer (Kandori et al., 2010; Kesten, 2010; Kesten and Kurino, 2013; Akahoshi, 2014). However, such domains have not been studied in the object assignment model with money transfer for non-quasi-linear preferences. Our paper is the first one that studies the common-tiered-object domain with money transfer.

The remaining parts are organized as follows. Section 2 introduces concepts and establishes the model. Section 3 defines the minimum price equilibria. Section 4 provides characterizations. Section 5 gives concluding remarks. All proofs are placed in the Appendix.

\section{The model and definitions}

Consider an economy with $n \geq 2$ agents and $m \geq 1$ objects. Denote the set of agents by $N \equiv\{1,2, \cdots, n\}$ and the set of (real) objects by $M \equiv\{1,2, \cdots, m\}$. Not receiving an object is called receiving a null object. We call it object 0 . Let $L \equiv M \cup\{0\}$. Each agent receives at most one object. We denote the object that agent $i \in N$ receives by $x_{i} \in L$. We denote the

\footnotetext{
${ }^{5}$ Precisely speaking, when objects are heterogenous, the Vickrey rule is equivalent to the minimum price rule for the quasi-linear preferences (Leonard, 1983). But these two rules are distinct for non-quasi-linear preferences (Morimoto and Serizawa, 2015).
} 
amount that agent $i$ pays by $t_{i} \in \mathbb{R}$. The agents' common consumption set is $L \times \mathbb{R}$, and a generic (consumption) bundle for agent $i$ is a pair $z_{i} \equiv\left(x_{i}, t_{i}\right) \in L \times \mathbb{R}$. Let $\mathbf{0} \equiv(0,0)$.

Each agent $i$ has a complete and transitive preference $R_{i}$ over $L \times \mathbb{R}$. Let $P_{i}$ and $I_{i}$ be the strict and indifference relations associated with $R_{i}$. A generic class of preferences is denoted by $\mathcal{R}$. We call $(\mathcal{R})^{n}$ a domain.

The following are basic properties of preferences, which we assume throughout the paper:

Money monotonicity: For each $x_{i} \in L$ and each pair $t_{i}, t_{i}^{\prime} \in \mathbb{R}$, if $t_{i}<t_{i}^{\prime},\left(x_{i}, t_{i}\right) P_{i}\left(x_{i}, t_{i}^{\prime}\right)$.

Object monotonicity: For each $x_{i} \in M$ and each $t_{i} \in \mathbb{R},\left(x_{i}, t_{i}\right) P_{i}\left(0, t_{i}\right)$.

Possibility of compensation: For each $t_{i} \in \mathbb{R}$ and each pair $x_{i}, x_{j} \in L$, there is a pair $t_{j}, t_{j}^{\prime} \in \mathbb{R}$ such that $\left(x_{i}, t_{i}\right) R_{i}\left(x_{j}, t_{j}\right)$ and $\left(x_{j}, t_{j}^{\prime}\right) R_{i}\left(x_{i}, t_{i}\right)$.

Continuity: For each $z_{i} \in L \times \mathbb{R}$, the upper contour set at $z_{i}, U C\left(R_{i}, z_{i}\right) \equiv\left\{z_{i}^{\prime} \in L \times \mathbb{R}\right.$ : $\left.z_{i}^{\prime} R_{i} z_{i}\right\}$ and the lower contour set at $z_{i}, L C\left(R_{i}, z_{i}\right) \equiv\left\{z_{i}^{\prime} \in L \times R: z_{i} R_{i} z_{i}^{\prime}\right\}$, are closed.

A preference $R_{i}$ is classical if it satisfies the four properties just defined. Let $\mathcal{R}^{C}$ be the class of classical preferences. We call $\left(\mathcal{R}^{C}\right)^{n}$ the classical domain.

Note that by money monotonicity, the possibility of compensation and continuity, for each $R_{i} \in \mathcal{R}^{C}$, each $z_{i} \in L \times \mathbb{R}$ and each $y \in L$, there is a unique amount $V_{i}\left(y ; z_{i}\right) \in \mathbb{R}$ such that $\left(y, V_{i}\left(y ; z_{i}\right)\right) I_{i} z_{i}$. We call $V_{i}\left(y ; z_{i}\right)$ the valuation of $y$ at $z_{i}$ for $R_{i}$.

An object allocation is an $n$-tuple $\left(x_{1}, \ldots, x_{n}\right) \in L^{n}$ such that for each pair $i, j \in N$, if $x_{i} \neq$ 0 and $i \neq j$, then $x_{i} \neq x_{j}$. We denote the set of object allocations by $X$. A (feasible) allocation is an $n$-tuple $z \equiv\left(z_{1}, \ldots, z_{n}\right) \equiv\left(\left(x_{1}, t_{1}\right), \ldots,\left(x_{n}, t_{n}\right)\right) \in[L \times \mathbb{R}]^{n}$ such that $\left(x_{1}, \ldots, x_{n}\right) \in X$. We denote the set of feasible allocations by $Z$. Given $z \in Z$, we denote its object and payment components at $z$ by $x \equiv\left(x_{1}, \ldots, x_{n}\right)$ and $t \equiv\left(t_{1}, \ldots, t_{n}\right)$, respectively.

A preference profile is an $n$-tuple $R \equiv\left(R_{i}\right)_{i \in N} \in \mathcal{R}^{n}$. Given $R \in \mathcal{R}^{n}$ and $N^{\prime} \subseteq N$, let $R_{N^{\prime}} \equiv\left(R_{i}\right)_{i \in N^{\prime}}$ and $R_{-N^{\prime}} \equiv R_{N \backslash N^{\prime}} \equiv\left(R_{i}\right)_{i \in N \backslash N^{\prime}}$.

Next, we introduce two properties of domains we focus on. First is "common-objectranking". It says that objects are ranked linearly, and for each payment, each agent prefers the bundle consisting of the object that has the higher rank and that payment to the bundle consisting of the object that has the lower rank and that payment. Let $\pi \equiv(\pi(1), \ldots, \pi(m), \pi(m+1))$ be a permutation of objects in $L$, where $\pi(1)$ denotes the object ranked first, $\pi(2)$ denotes the object ranked second, and so on. For every pair $x, y \in L, x>_{\pi} y$ means that $x$ has a higher rank than $y$ according to $\pi$.

A preference $R_{i} \in \mathcal{R}^{C}$ ranks objects according to $\pi$ if for each $x_{i} \in L$ and each $t_{i} \in \mathbb{R}$, $\left(\pi(1), t_{i}\right) P_{i}\left(\pi(2), t_{i}\right) \cdots P_{i}\left(\pi(m), t_{i}\right) P_{i}\left(\pi(m+1), t_{i}\right)$.

Remark 1: Since $R_{i} \in \mathcal{R}^{C}$, object monotonicity implies $\pi(m+1)=0$.

Figure 1 illustrates a preference $R_{i}$ ranking objects according to $\pi$ for $M=\{A, B, C\}$ and $\pi=(\pi(1), \pi(2), \pi(3), \pi(4))=(C, B, A, 0)$. 


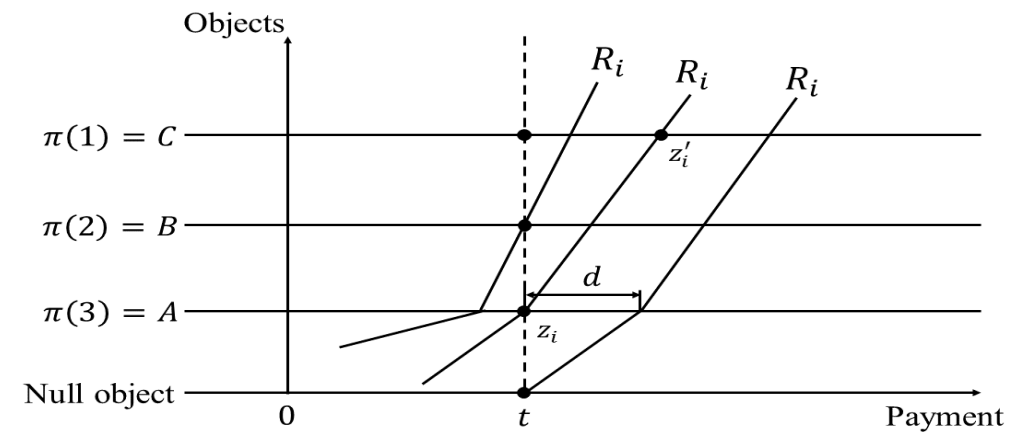

\section{Figure 1 Illustration of preference ranking objects according to $\pi$}

In Figure 1, there are four horizontal lines. The bottom line corresponds to the null object, the middle two lines to objects $A$ and $B$, and the top line to object $C$, respectively. The intersection of the vertical line and each horizontal line denotes the bundle consisting of the corresponding object and no payment. For example, the origin $\mathbf{0}$ denotes the bundle consisting of the null object and no payment. For each point on one of three horizontal lines, the distance from that point to the vertical line denotes the payment. For example, $z_{i}$ denotes the bundle consisting of object $A$ and payment $t$. By money monotonicity, moving rightward along the same line makes the agent worse off, i.e., if $d>0$, then $(A, t) P_{i}(A, t+d)$. If the bundles are connected by a indifference curve, for example, $z_{i}$ and $z_{i}^{\prime}$, it means that agent $i$ is indifferent between them, i.e., $z_{i} I_{i} z_{i}^{\prime}$. In Figure 1 , for each $t \in \mathbb{R},(C, t) P_{i}(B, t) P_{i}(A, t) P_{i}(0, t)$ and $R_{i}$ ranks objects according to $\pi=(C, B, A, 0)$.

Let $\mathcal{R}^{R}(\pi)$ be the class of preferences ranking objects according to $\pi$ and note that $\mathcal{R}^{R}(\pi) \varsubsetneqq$ $\mathcal{R}^{C}$. A preference profile $R$ ranks objects according to $\pi$ if each preference in the preference profile all ranks objects according to $\pi$, i.e., for each $i \in N, R_{i} \in \mathcal{R}^{R}(\pi)$.

Figure 2 illustrates the preference profile $R$ ranking objects according to $\pi$ for $N=\{1,2\}$, $M=\{A, B, C\}$, and $\pi=(\pi(1), \pi(2), \pi(3), \pi(4))=(C, B, A, 0)$.

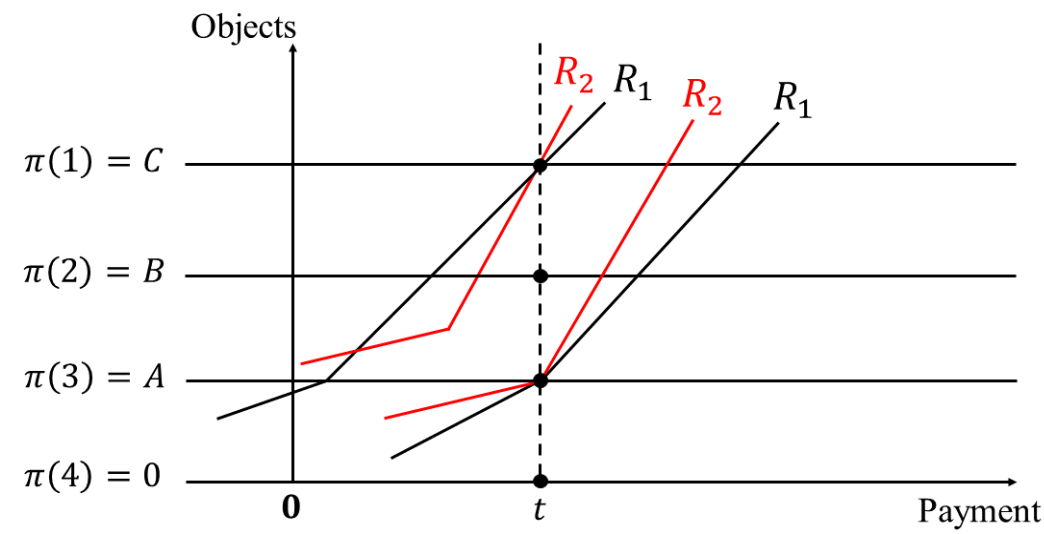

Figure 2 Illustration of preference profile ranking objects according to $\pi$ 
In Figure 2 , for each $t \in \mathbb{R},(C, t) P_{1}(B, t) P_{1}(A, t) P_{1}(0, t)$ and $(C, t) P_{2}(B, t) P_{2}(A, t) P_{2}(0, t)$. Thus, $R$ ranks objects according to $\pi$.

We call $\left(\mathcal{R}^{R}(\pi)\right)^{n}$ the common-object-ranking domain if there is an object permutation $\pi$ such that for each $R \in\left(\mathcal{R}^{R}(\pi)\right)^{n}, R$ ranks object according to $\pi$.

Second is "common-tiered-object ranking". It says that objects are partitioned into tiers, and for each payment, each agent prefers the bundle consisting of the object in the higher tier and that payment to the bundle consisting of the object in the lower tier and that payment. We describe a tier partition by an indexed family $\mathcal{T}=\left\{T_{l}\right\}_{l \in K}$ of non-empty subsets of $L$ such that (i) $K \equiv\{1,2, \cdots, k\}$ and $1 \leq k \leq m+1$, (ii) $\cup_{l \in K} T_{l}=L$ and (iii) for each $l, l^{\prime} \in K$ with $l \neq l^{\prime}, T_{l} \cap T_{l^{\prime}}=\varnothing$, where $T_{l}$ denotes the $l$-th tier for each $l \in K$. For every pair $x, y \in L$, $x>_{\mathcal{T}} y$ means that $x$ is in a higher tier than $y$ according to $\mathcal{T}$.

A preference $R_{i} \in \mathcal{R}^{C}$ ranks object according to $\mathcal{T}$ if for each $t_{i} \in \mathbb{R}$, each $x \in T_{l}$ and each $y \in T_{l^{\prime}}$ with $l \neq l^{\prime}$ and $l<l^{\prime},\left(x, t_{i}\right) P_{i}\left(y, t_{i}\right)$.

Remark 2: (i) Since $R_{i} \in \mathcal{R}^{C}$, object monotonicity implies $k \geq 2$ and $T_{k}=\{0\}$.

(ii) If a preference $R_{i} \in \mathcal{R}^{C}$ ranks objects according to $\pi$, then $R_{i}$ also ranks objects according to $\mathcal{T}$ such that $T_{1}=\{\pi(1)\}, T_{2}=\{\pi(2)\}, \cdots, T_{m+1}=\{\pi(m+1)\}$.

Figure 3 illustrates a preference $R_{i}$ ranking objects according to $\mathcal{T}$ for $M=\{A, B, C\}$ and $\mathcal{T}=T_{1} \cup T_{2} \cup T_{3}$ with $T_{1}=\{B, C\}, T_{2}=\{A\}$, and $T_{3}=\{0\}$.

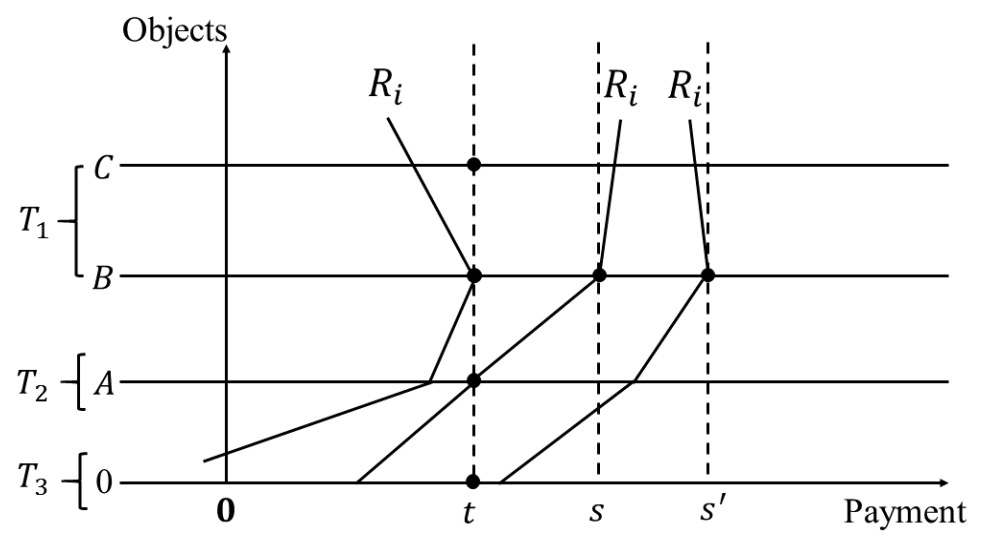

Figure 3 Illustration of preference ranking objects according to $\mathcal{T}$

In Figure 3, for each $t \in \mathbb{R}$, each $y \in T_{1}$, each $x \in T_{2}$, namely $x=A$, and $0 \in T_{3}$, we have $(y, t) P_{i}(A, t) P_{i}(0, t)$. Note that $(C, s) P_{i}(B, s) P_{i}(0, t)$ and $\left(B, s^{\prime}\right) P_{i}\left(C, s^{\prime}\right) P_{i}(0, t)$. Thus, $R_{i}$ ranks objects according to $\mathcal{T}$, but does not rank objects according to any object permutation.

Let $\mathcal{R}^{T}(\mathcal{T})$ be the class of preferences ranking objects according to $\mathcal{T}$. Obviously, $\mathcal{R}^{T}(\mathcal{T}) \subset$ $\mathcal{R}^{C}$. A preference profile $R$ ranks objects according to $\mathcal{T}$ if each preference in the profile all ranks objects according to $\mathcal{T}$, i.e., for each $i \in N, R_{i} \in \mathcal{R}^{T}(\mathcal{T})$.

Figure 4 illustrates the preference profile $R$ ranking objects according to $\mathcal{T}$ for $N=\{1,2\}$, $M=\{A, B, C\}$, and $\mathcal{T}=T_{1} \cup T_{2} \cup T_{3}$ with $T_{1}=\{B, C\}, T_{2}=\{A\}$, and $T_{3}=\{0\}$. 


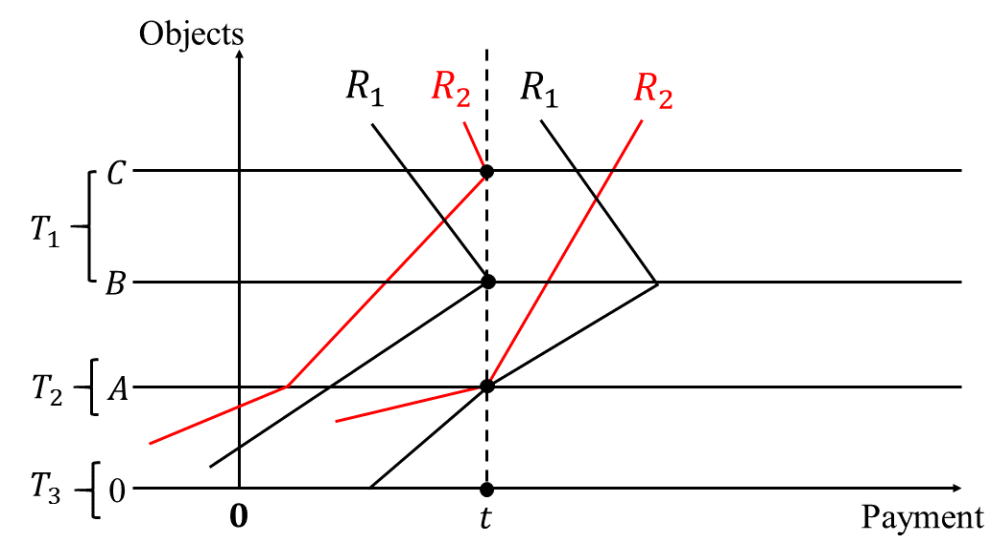

Figure 4 Illustration of preference profile ranking objects according to $\mathcal{T}$

In Figure 4 , for each $t \in \mathbb{R},(C, t) P_{1}(B, t) P_{1}(A, t) P_{1}(0, t)$ and $(B, t) P_{2}(C, t) P_{2}(A, t) P_{2}(0, t)$. Thus, $R$ ranks objects according to $\mathcal{T}$.

We call $\left(\mathcal{R}^{T}(\mathcal{T})\right)^{n}$ the common-tiered-object domain if there is an indexed family $\mathcal{T}=$ $\left\{T_{i}\right\}_{i \in K}$ such that for each $R \in \mathcal{R}^{T}(\mathcal{T})^{n}, R$ ranks objects according to $\mathcal{T}$.

Remark 3: (i) Any common-object-ranking domain is included in the common-tiered-object domain with respect to some $\mathcal{T}=\left\{T_{i}\right\}_{i \in K}$. If $2 \leq k<m+1$, such a inclusion relation is strict. (ii) If $k=m+1$, a common-tiered-object domain with $k$ tiers is a common-object-ranking domain.

(iii) Consider two common-tiered-object domains with respect to $\mathcal{T}=\left\{T_{l}\right\}_{l \in K}$ and $\mathcal{T}^{\prime}=$ $\left\{T_{l^{\prime}}^{\prime}\right\}_{l^{\prime} \in K^{\prime}}$. If $\mathcal{T}^{\prime}$ is coarser than $\mathcal{T}$, then the common-tiered-object domain with respect to $\mathcal{T}$ is a subset of the one with respect to $\mathcal{T}^{\prime}{ }^{6}$

(iv) Since the classical domain is the common-tiered-object domain with the coarsest class of tiers, that is, $k=2$, any common-tiered-object domain is a subset of the classical domain.

An (allocation) rule on $\mathcal{R}^{n}$ is a mapping $f$ from $\mathcal{R}^{n}$ to $Z$. Given a rule $f$ and $R \in \mathcal{R}^{n}$, we denote bundle assigned to agent $i$ by $f_{i}(R) \equiv\left(x_{i}(R), t_{i}(R)\right)$ where $x_{i}(R)$ denotes the assigned object and $t_{i}(R)$ the associated payment. We write,

$$
f(R) \equiv\left(f_{i}(R)\right)_{i \in N}, x(R) \equiv\left(x_{i}(R)\right)_{i \in N}, \text { and } t(R) \equiv\left(t_{i}(R)\right)_{i \in N} .
$$

Now, we introduce standard properties of rules. An allocation $z \equiv\left(\left(x_{i}, t_{i}\right)\right)_{i \in N} \in Z$ is (Pareto-)efficient for $R \in \mathcal{R}^{n}$ if there is no feasible allocation $z^{\prime} \equiv\left(\left(x_{i}^{\prime}, t_{i}^{\prime}\right)\right)_{i \in N} \in Z$ such that ${ }^{7}$

(i) for each $i \in N, z_{i}^{\prime} R_{i} z_{i}$, (ii) for some $j \in N, z_{j}^{\prime} P_{j} z_{j}$, and (iii) $\sum_{i \in N} t_{i}^{\prime} \geq \sum_{i \in N} t_{i}$.

For each preference profile, the rule chooses an efficient allocation.

Efficiency: For each $R \in \mathcal{R}^{n}, f(R)$ is efficient for $R$.

\footnotetext{
${ }^{6} \mathcal{T}^{\prime}$ is coarser than $\mathcal{T}$ if for each $l \in K$, there is $l^{\prime} \in K^{\prime}$ such that $T_{l} \subseteq T_{l^{\prime}}$.

${ }^{7}$ Efficiency described here takes the perspective of object suppliers, i.e., governments and auctioneers. Object suppliers only care about their revenues.
} 
No agent ever benefits from misrepresenting his preference.

Strategy-proofness: For each $R \in \mathcal{R}^{n}$, each $i \in N$ and each $R_{i}^{\prime} \in \mathcal{R}, f_{i}\left(\stackrel{\text { truth }}{R_{i}}, R_{-i}\right) \stackrel{\text { truth }}{R_{i}} f_{i}\left(\stackrel{\text { lie }}{R_{i}^{\prime}}, R_{-i}\right){ }^{8}$

No agent is ever assigned a bundle that makes him worse off than he would be if he had received the null object and paid nothing.

Individual rationality: For each $R \in \mathcal{R}^{n}$ and each $i \in N, f_{i}(R) R_{i} \mathbf{0}$.

The payment of each agent is always nonnegative.

No subsidy: For each $R \in \mathcal{R}^{n}$ and each $i \in N, t_{i}(R) \geq 0$.

The final property is a weak variant of no subsidy: if an agent receives the null object, his payment is nonnegative.

No subsidy for losers: For each $R \in \mathcal{R}^{n}$, if $x_{i}(R)=0, t_{i}(R) \geq 0$.

\section{The Minimum price equilibria}

In this section, we define the equilibria and minimum price equilibria, and state several facts related to them. Throughout the section, let us fix $\mathcal{R} \subseteq \mathcal{R}^{C}$ and obviously, all facts hold on the common-object-ranking and common-tiered-object domains.

\subsection{Definitions of equilibria and minimum price equilibria}

Let $p \equiv\left(p_{1}, \cdots, p_{m}\right) \in \mathbb{R}_{+}^{m}$ be a price vector. The budget set at $p$ is defined as $B(p) \equiv$ $\left\{\left(x, p_{x}\right): x \in L\right\}$, where $p_{x}=0$ if $x=0$. Given $R_{i} \in \mathcal{R}$, the demand set at $p$ for $R_{i}$ is defined as $D\left(R_{i}, p\right) \equiv\left\{x \in L:\right.$ for each $\left.y \in L,\left(x, p_{x}\right) R_{i}\left(y, p_{y}\right)\right\}$.

Definition: Let $R \in \mathcal{R}^{n}$. A pair $((x, t), p) \in Z \times \mathbb{R}_{+}^{m}$ is a (Walrasian) equilibrium for $R$ if

$$
\begin{aligned}
\text { for each } i & \in N, x_{i} \in D\left(R_{i}, p\right) \text { and } t_{i}=p_{x_{i}}, \\
\text { for each } y & \in M \text {, if for each } i \in N, x_{i} \neq y \text {, then } p_{y}=0 .
\end{aligned}
$$

Condition (E-i) says that each agent receives an object from his demand set and pays its price. Condition (E-ii) says that the prices of unassigned objects are zero.

Fact 1 (Alkan and Gale, 1990; Alaei et al, 2016) (Existence). For each $R \in \mathcal{R}^{n}$, there is an equilibrium.

Given $R \in \mathcal{R}^{n}$, we denote the set of equilibria for $R$ by $W(R)$, the set of equilibrium allocations for $R$ by $Z(R)$, and the set of equilibrium price vectors for $R$ by $P(R)$, respectively, i.e.,

$$
\begin{aligned}
& Z(R) \equiv\left\{z \in Z: \text { for some } p \in \mathbb{R}_{+}^{m},(z, p) \in W(R)\right\}, \text { and } \\
& P(R) \equiv\left\{p \in \mathbb{R}_{+}^{m}: \text { for some } z \in Z,(z, p) \in W(R)\right\} .
\end{aligned}
$$

${ }^{8}$ Thomson (2015) introduces this notation. 
Fact 2 (Demange and Gale, 1985) (Lattice property). For each $R \in \mathcal{R}^{n}, P(R)$ is a complete lattice and there is a unique equilibrium price vector $p \in P(R)$ such that for each $p^{\prime} \in P(R)$, $p \leq p^{\prime}$.

A minimum price equilibrium (MPE) is an equilibrium whose price vector is minimum. Given $R \in \mathcal{R}^{n}$, let $p^{\min }(R)$ be the minimum equilibrium price vector for $R, W^{\min }(R)$ the set of minimum price equilibria associated with $p^{\min }(R)$ and $Z^{\min }(R)$ the set of minimum price equilibrium allocations associated with $p^{\min }(R)$, respectively, i.e.,

$$
Z^{\min }(R) \equiv\left\{z \in Z:\left(z, p^{\min }(R)\right) \in W^{\min }(R)\right\} .
$$

Although there might be several minimum price equilibria, they are indifferent for each agent, i.e., for each $R \in \mathcal{R}^{n}$, each pair $z, z^{\prime} \in Z^{\min }(R)$ and each $i \in N, z_{i} I_{i} z_{i}^{\prime}$.

\subsection{Illustrations of minimum price equilibria}

In this subsection, we illustrate the definition of minimum price equilibrium for $\mathcal{R} \subseteq \mathcal{R}^{C}$, $\mathcal{R} \subseteq \mathcal{R}^{R}(\pi)$ and $\mathcal{R} \subseteq \mathcal{R}^{T}(\mathcal{T})$ by means of three figures. Since $R$ is fixed, we write $p^{\text {min }}$ instead of $p^{\min }(R)$ for illustrations.

Figure 5 illustrates a MPE for $\mathcal{R} \subseteq \mathcal{R}^{C}, N=\{1,2,3\}$, and $M=\{A, B, C, D\}$.

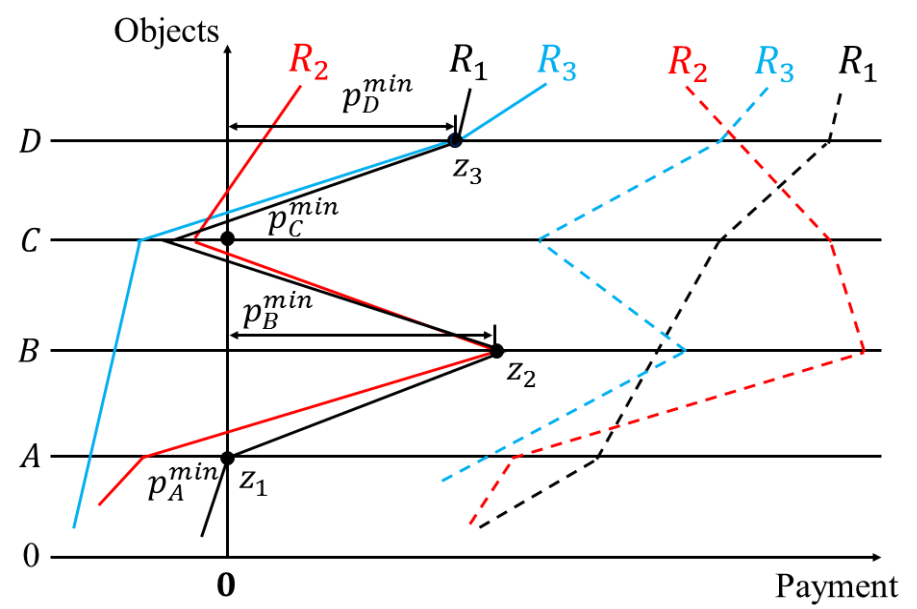

Figure 5 Illustration of minimum price equilibrium for preference profile from classical domain

In Figure 5, a MPE allocation is as follows: agent 1 receives object $A$ and pays 0 . Agent 2 receives object $B$ and pays $p_{B}^{\min }$. Agent 3 receives object $D$ and pays $p_{D}^{\min }$. The prices of objects $A$ and $C$ are 0 .

Let's see why the allocation $z \equiv\left(z_{1}, z_{2}, z_{3}\right)$ is a MPE allocation. First, for each agent $i=1,2,3, z_{i}$ is maximal for $R_{i}$ in the budget set $\left\{\mathbf{0},\left(A, p_{A}^{\min }\right),\left(B, p_{B}^{\min }\right),\left(C, p_{C}^{\min }\right),\left(D, p_{D}^{\min }\right)\right\}$. Thus, $z$ is an equilibrium allocation. Let $p^{\min } \equiv\left(p_{A}^{\min }, p_{B}^{\min }, p_{C}^{\min }, p_{D}^{\min }\right)$.

Next, let $p \equiv\left(p_{A}, p_{B}, p_{C}, p_{D}\right)$ be an equilibrium price. We show $p \geq p^{\text {min }}$. By the nonnegativity of prices, $p_{A} \geq 0=p_{A}^{\min }$ and $p_{C} \geq 0=p_{C}^{\mathrm{min}}$. 
If $p_{B}<p_{B}^{\min }$ and $p_{D}<p_{D}^{\min }$, then by $p_{A} \geq 0$ and $p_{C} \geq 0$, all three agents prefer $\left(B, p_{B}\right)$ or $\left(D, p_{D}\right)$ to $\mathbf{0},\left(A, p_{A}\right)$ and $\left(C, p_{C}\right)$. In such a case, at least one agent cannot receive an object from his demand set, contradicting (E-i). Thus, $p_{B} \geq p_{B}^{\min }$ or $p_{D} \geq p_{D}^{\min }$.

If $p_{B}<p_{B}^{\min }$, then $p_{D} \geq p_{D}^{\min }$. By $p_{A} \geq 0$ and $p_{C} \geq 0$, both agents 1 and 2 prefer $\left(B, p_{B}\right)$ to $\mathbf{0},\left(A, p_{A}\right),\left(C, p_{C}\right)$ and $\left(D, p_{D}\right)$. In such a case, one of agents 1 and 2 cannot receive the object he demands, contradicting (E-i). Thus, $p_{B} \geq p_{B}^{\min }$.

If $p_{D}<p_{D}^{\min }$, then by $p_{A} \geq 0, p_{B} \geq p_{B}^{\min }$ and $p_{C} \geq 0$, both agents 1 and 3 prefer $\left(D, p_{D}\right)$ to $\mathbf{0},\left(A, p_{A}\right),\left(B, p_{B}\right)$ and $\left(C, p_{C}\right)$. In such a case, one of agents 1 and 3 cannot receive the object he demands, contradicting (E-i). Thus, $p_{D} \geq p_{D}^{\min }$. Thus, $p \geq p^{\min }$ and $\left(z, p^{\min }\right)$ is a MPE.

In Figure 5, the minimum equilibrium prices may not be monotonic with respect to the object rankings and which objects are unassigned depends on the preference profile we choose. However, when $\mathcal{R} \subseteq \mathcal{R}^{R}(\pi)$ or $\mathcal{R} \subseteq \mathcal{R}^{T}(\mathcal{T})$, the minimum equilibrium prices are monotonic with respect to the object rankings or object-tier rankings. Unassigned objects are the ones that have the lower ranks or are in the lower tiers. We specify these features in the following.

Figure 6 illustrates the MPE for $\mathcal{R} \subseteq \mathcal{R}^{R}(\pi), N=\{1,2,3\}, M=\{A, B, C, D\}$, and $\pi=(D, C, B, A, 0)$.

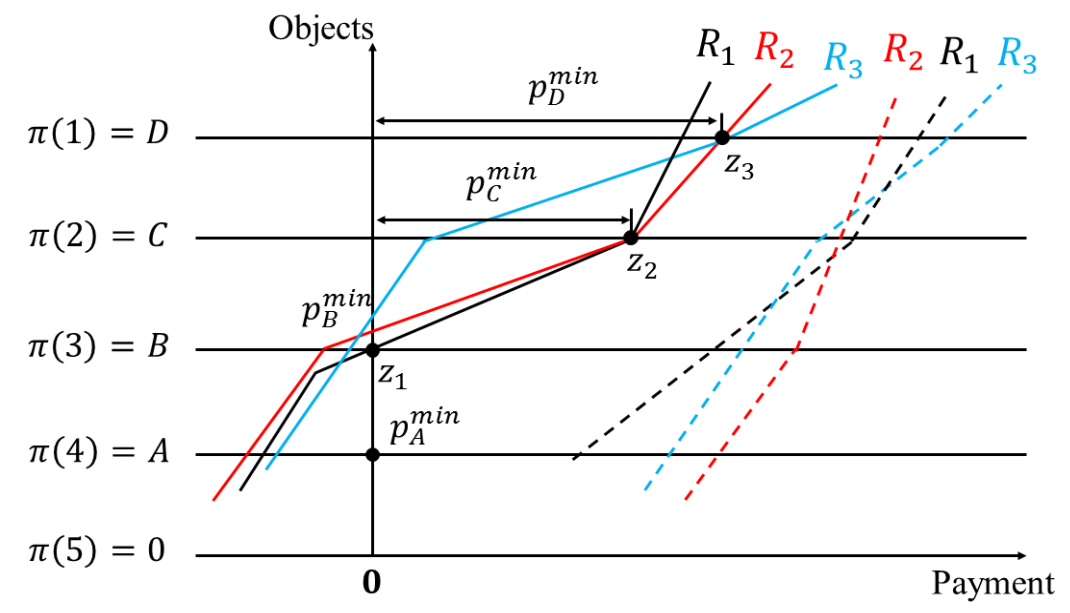

\section{Figure 6 Illustration of minimum price equilibrium for preference profile from common-object-ranking domain}

Similarly to Figure $5,\left(z, p^{\min }\right)$ is a MPE in Figure 6. Note that $p_{D}^{\min }>p_{C}^{\min }>p_{B}^{\min }=p_{D}^{\min }$. For $\mathcal{R} \subseteq \mathcal{R}^{R}(\pi)$, the minimum equilibrium price of object that has the higher rank is larger than that of object that has the lower rank. This feature is summrized as Remark 4.

Remark 4: Let $\mu \equiv \min \{n, m+1\}$. In the MPE for $\mathcal{R} \subseteq \mathcal{R}^{R}(\pi)$, (i) if $m+1 \leq n$, then $\mu=m+1, p_{\pi(1)}^{\min }(R)>\cdots>p_{\pi(m)}^{\min }(R)>p_{\pi(\mu)}^{\min }(R)=0$, and all the objects are assigned, and (ii) if $m+1>n$, then $\mu=n, p_{\pi(1)}^{\min }(R)>\cdots>p_{\pi(\mu)}^{\min }(R)=\cdots=p_{\pi(m)}^{\min }(R)=0$ and objects ranked lower than $\pi(\mu)$ are unassigned. ${ }^{9}$

\footnotetext{
${ }^{9}$ To see (i), let $m+1 \leq n$. Then, $\mu=m+1$ implies $\pi(\mu)=0$ and $p_{\pi(\mu)}^{\min }(R)=0 . \quad \mu=m+1$ also
} 
Figure 7 illustrates the MPE for $\mathcal{R} \subseteq \mathcal{R}^{T}(\mathcal{T}), N=\{1,2,3\}, M=\{A, B, C, D\}$, and $\mathcal{T}=T_{1} \cup T_{2} \cup T_{3}$ with $T_{1}=\{C, D\}, T_{2}=\{A, B\}$, and $T_{3}=\{0\}$.

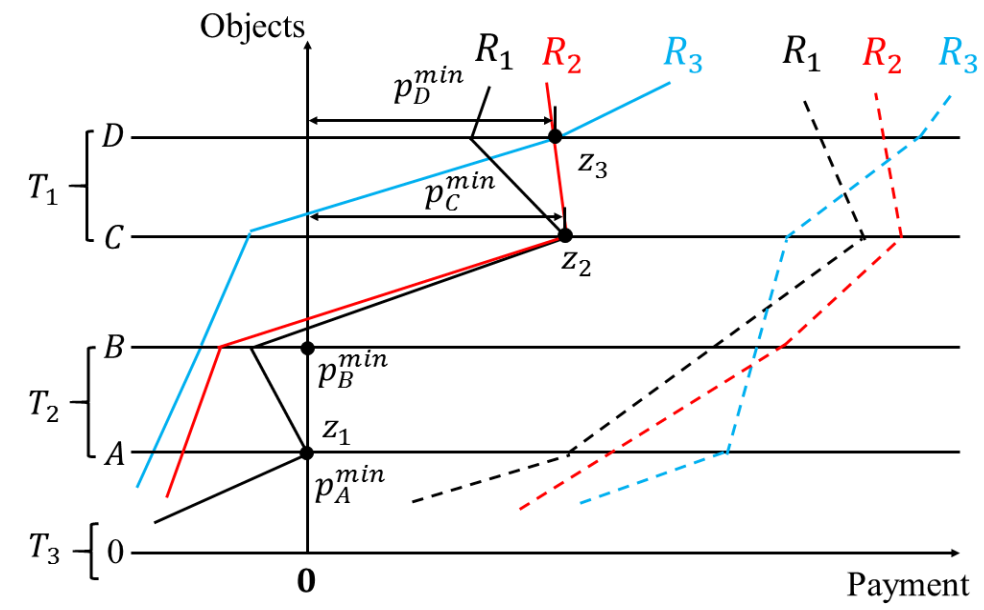

\section{Figure 7 Illustration of minimum price equilibrium for preference profile from common-tiered-object domain}

Similarly to Figure $5,\left(z, p^{\min }\right)$ is a MPE in Figure 7 . Note that $\min \left\{p_{C}^{\min }, p_{D}^{\min }\right\}>p_{A}^{\min }=$ $p_{B}^{\min }=0$. In the MPE for $\mathcal{R} \subseteq \mathcal{R}^{T}(\mathcal{T})$, the prices of the objects in higher tiers are larger than those in lower tiers. Remark 5 is parrell to Remark 4.

Remark 5: Let $\mu \equiv \min \{n, m+1\}$. Let $l_{0} \in K$ be such that $\sum_{l=1}^{l_{0}-1}\left|T_{l}\right|<\mu \leq \sum_{l=1}^{l_{0}}\left|T_{l}\right|{ }^{10}$ In the MPE for $\mathcal{R} \subseteq \mathcal{R}^{T}(\mathcal{T})$, (i) if $l<l_{0}$, for each $x \in T_{l}, p_{x}^{\min }(R)>0$ and $x$ is assigned to some agent, (ii) if $l<l^{\prime} \leq l_{0}, \min \left\{p_{x}^{\min }(R): x \in T_{l}\right\}>\max \left\{p_{x}^{\min }(R): x \in T_{l^{\prime}}\right\}$, (iii) there is $x \in T_{l_{0}}$ such that $p_{x}^{\min }(R)=0$ and $x$ is assigned to some agent, and (iv) if $l>l_{0}$, for each $x \in T_{l}$, $p_{x}^{\min }(R)=0$ and $x$ is unassigned..$^{11}$

implies that there is $i \in N$ such that $x_{i}=0$. If there is $x \in M$ such that $p_{x}^{\min }(R)=0$, then $\left(x, p_{x}^{\min }(R)\right) P_{i} \mathbf{0}$, contradicting $0 \in D\left(R_{i}, p^{\min }(R)\right)$. Thus, for each $x \in M$, we have $p_{x}^{\min }(R)>0$. Thus, by (E-ii), all the objects are assigned. To see $p_{\pi(1)}^{\min }(R)>\cdots>p_{\pi(m)}^{\min }(R)>0$, by contradiction, suppose that there is a pair $x, y \in M$ such that $y>_{\pi} x \geq_{\pi} \pi(\mu)$ and $p_{x}^{\min }(R) \geq p_{y}^{\min }(R)$. Let $j \in N$ be such that $x_{j}=x$. By (E-i), we have $x \in D\left(R_{j}, p^{\min }(R)\right)$. By $R_{j} \in \mathcal{R}^{R}(\pi)$, we have $\left(y, p_{y}^{\min }(R)\right) P_{j}\left(x, p_{x}^{\min }(R)\right)$, contradicting $x \in D\left(R_{j}, p^{\min }(R)\right)$. Thus $0=p_{\pi(\mu)}^{\min }(R)<p_{\pi(\mu-1)}^{\min }(R)<\cdots<p_{\pi(1)}^{\min }(R)$.

To see (ii), let $m+1 \geq n$. Then, $\mu=n$. If there is a pair $x, y \in M$ such that $x \geq_{\pi} \pi(\mu)$ and $x$ is unassinged, and $y<_{\pi} \pi(\mu)$ and $y$ is assigned to some $i \in N$, then, by (E-i), we have $y \in D\left(R_{i}, p^{\min }(R)\right)$. By (E-ii), we have $p_{x}^{\min }(R)=0$. Thus, by $R_{i} \in \mathcal{R}^{R}(\pi)$, we have $\left(x, p_{x}^{\min }(R)\right) P_{i}\left(y, p_{y}^{\min }(R)\right)$, contradicting $y \in D\left(R_{i}, p^{\min }(R)\right)$. Thus, for each $x \in M$ such that $x \geq_{\pi} \pi(\mu), x$ is assigned to some agent. By $\mu=n$, objects ranked lower than $\pi(\mu)$ are unassigned. Thus, by (E-ii), we have $p_{\pi(\mu+1)}^{\min }(R)=\cdots=p_{\pi(m)}^{\min }(R)=0$. Similarly to (i), we can show $p_{\pi(1)}^{\min }(R)>\cdots>p_{\pi(\mu)}^{\min }(R) \geq p_{\pi(\mu+1)}^{\min }(R)=0$. If $p_{\pi(\mu)}^{\min }(R)>0$, then $\{\pi(1), \cdots, \pi(\mu)\}$ is weakly underdemanded, contradicting Fact 3. Thus $p_{\pi(\mu)}^{\min }(R)=0$.

${ }^{10}$ If $l_{0}=1$, then $\sum_{l=1}^{l_{0}-1}\left|T_{l}\right|=0$.

${ }^{11}$ To see (i), let $l<l_{0}$. By contradiction, suppose there is $x \in T_{l}$ such that $p_{x}^{\min }(R)=0$. Then, by $l<l_{0}$, $\sum_{l^{\prime}=1}^{l_{0}-1}\left|T_{l^{\prime}}\right|<\mu \leq n,(\mathrm{E}-\mathrm{i})$, and (E-ii), there is $y \in M$ and $i \in N$ such that $y<\mathcal{T} x$ and $y \in D\left(R_{i}, p^{\min }(R)\right)$. By $p_{x}^{\min }(R)=0$ and $R_{i} \in \mathcal{R}^{T}(\mathcal{T})$, we have $\left(x, p^{\min }(R)\right) P_{i}\left(y, p^{\min }(R)\right)$, contradicting $y \in D\left(R_{i}, p^{\min }(R)\right)$. Thus, if 


\subsection{Overdemanded and underdemanded sets}

In the following, we define the concepts of an "overdemanded set" and a "(weakly) underdemanded set" to characterize minimum equilibrium price vector.

Given $p$ and $M^{\prime} \subseteq M$, let $N^{D}\left(p, M^{\prime}\right) \equiv\left\{i \in N: D\left(R_{i}, p\right) \subseteq M^{\prime}\right\}$ and $N^{W D}\left(p, M^{\prime}\right) \equiv\{i \in$ $\left.N: D\left(R_{i}, p\right) \cap M^{\prime} \neq \varnothing\right\}$.

Example 1. Figure 5 illustrates $N^{D}\left(p, M^{\prime}\right)$ and $N^{W D}\left(p, M^{\prime}\right)$ for $M^{\prime}=\{A\},\{A, B\}$ and $\{A, B, D\}$. For $M^{\prime}=\{A\}$, we have $N^{D}\left(p^{\min },\{A\}\right)=\varnothing$ and $N^{W D}\left(p^{\min },\{A\}\right)=\{1\}$. For $M^{\prime}=\{A, B\}$, we have $N^{D}\left(p^{\min },\{A, B\}\right)=\{2\}$ and $N^{W D}\left(p^{\min },\{A, B\}\right)=\{1,2\}$. For $M^{\prime}=$ $\{A, B, D\}$, we have $N^{D}\left(p^{\min },\{A, B, D\}\right)=\{1,2,3\}$ and $N^{W D}\left(p^{\min },\{A, B, D\}\right)=\{1,2,3\}$.

Given a set $S,|S|$ denotes the cardinality of $S$.

Definition: (i) A non-empty set $M^{\prime} \subseteq M$ of objects is overdemanded at $p$ for $R$ if $\left|N^{D}\left(p, M^{\prime}\right)\right|>\left|M^{\prime}\right|$.

(ii) A non-empty set $M^{\prime} \subseteq M$ of objects is (weakly) underdemanded at $p$ for $R$ if

$$
\left[\forall x \in M^{\prime}, p_{x}>0\right] \Rightarrow\left|N^{W D}\left(p, M^{\prime}\right)\right|(\leq)<\left|M^{\prime}\right|
$$

By using "overdemanded set" and "(weakly) underdemanded set", we can characterize the minimum equilibrium price vector.

Fact 3 (Morimoto and Serizawa, 2015). ${ }^{12}$ Let $R \in \mathcal{R}^{n}$. A price vector $p$ is a minimum equilibrium price vector for $R$ if and only if no set is overdemanded and no set is weakly underdemanded at $p$ for $R$.

Example 2. Figure 5 illustrates Fact 3. First, $\left|N^{D}\left(p^{\min },\{A\}\right)\right|=0<|\{A\}|=1$ and $\left|N^{D}\left(p^{\min },\{C\}\right)\right|=0<|\{C\}|=1$. Similarly, $\{B\}$ nor $\{D\}$ are overdemanded. Then, $\left|N^{D}\left(p^{\min },\{A, B\}\right)\right|=1<|\{A, B\}|=2$ and $\left|N^{D}\left(p^{\min },\{A, C\}\right)\right|=0<|\{A, C\}|=2$. Similarly,

$l<l_{0}$, for each $x \in T_{l}, p_{x}^{\min }(R)>0$. By (E-ii), $x$ is assigned to some agent.

To see (ii), let $l<l^{\prime} \leq l_{0}, x \in T_{l}$, and $y \in T_{l^{\prime}}$ be such that $p_{x}^{\min }(R)=\min \left\{p_{x}^{\min }(R): x \in T_{l}\right\}$ and $p_{y}^{\min }(R)=\max \left\{p_{x}^{\min }(R): x \in T_{l^{\prime}}\right\}$. By contradiction, suppose $p_{x}^{\min }(R) \leq p_{y}^{\min }(R)$. By (i) and $l<l_{0}$, $0<p_{x}^{\min }(R) \leq p_{y}^{\min }(R)$. Thus, by (E-i) and (E-ii), there is $j \in N$ such that $y \in D\left(R_{j}, p^{\min }(R)\right)$. By $x>_{\mathcal{T}} y$, $R_{j} \in \mathcal{R}^{T}(\mathcal{T})$, and $p_{x}^{\min }(R) \leq p_{y}^{\min }(R)$, we have $\left(x, p^{\min }(R)\right) P_{j}\left(y, p^{\min }(R)\right)$, contradicting $y \in D\left(R_{j}, p^{\min }(R)\right)$. Thus, $p_{x}^{\min }(R)>p_{y}^{\min }(R)$.

To see (iii), if $n \geq m+1$, then $\mu=m+1$. Thus $T_{l_{0}}=\{0\}$ and $p_{0}^{\min }(R)=0$. By $n \geq m+1$, there is $i \in N$ such that $x_{i}=0$. If $n<m+1$, then $\mu=n$. By Fact 3, there is $x \in M$ such that $p_{x}^{\min }(R)=0$ and $x$ is assigned to some agent. Then there is $l \in K$ such that $x \in T_{l}$, and by (i), $l \geq l_{0}$. If $l>l_{0}$, then, for each $x \in T_{l_{0}}, p_{x}^{\min }(R)>0$. If not, then there is $y \in T_{l_{0}}$ such that $p_{y}^{\min }(R)=0$. Then, $\left(y, p^{\min }(R)\right) P_{i}\left(x, p^{\min }(R)\right)$, contradicting $x \in D\left(R_{i}, p^{\min }(R)\right)$. Let $l^{\prime \prime} \leq l_{0}$. Thus, for each $x \in T_{l^{\prime \prime}}, p_{x}^{\min }(R)>0$. However, by the definition of $l_{0}, \cup_{l \in\left\{1,2, \cdots, l_{0}\right\}} T_{l}$ is weakly underdemanded. This contradicts Fact 3 . Thus $l=l_{0}$.

To see (iv), let $l>l_{0}$. By contradiction, suppose there is $x \in T_{l}$ such that $p_{x}^{\min }(R)>0$. Then, by (Eii), there is $i \in N$ such that $x \in D\left(R_{i}, p^{\min }(R)\right)$. If there is $l^{\prime}<l$ and $y \in T_{l^{\prime}}$ such that $p_{y}^{\min }(R)=0$, then $\left(y, p^{\min }(R)\right) P_{i}\left(x, p^{\min }(R)\right)$, contradicting $x \in D\left(R_{i}, p^{\min }(R)\right)$. Thus, for each $l^{\prime}<l$ and each $y \in T_{l^{\prime}}$, $p_{y}^{\min }(R)>0$. However, this contradicts (iii). Thus, if $l>l_{0}$, for each $x \in T_{l}, p_{x}^{\min }(R)=0$. If there is $x \in T_{l}$ such that $x$ is assigned to some $i \in N$, then, by the definition of $l_{0}$ and $l>l_{0}$, there is $y>_{T} x$ such that $y$ is unassigned. Thus, by (E-ii), $p_{y}^{\min }(R)=0$. Then, $\left.y, p^{\min }(R)\right) P_{i}\left(x, p^{\min }(R)\right)$, contradicting $x \in D\left(R_{i}, p^{\min }(R)\right)$. Thus, if $l>l_{0}$, for each $x \in T_{l}, x$ is unassigned.

${ }^{12}$ Mishra and Talman (2010) establish the parallel characterization for quasi-linear preferences. 
$\{A, D\},\{B, D\},\{B, C\}$ nor $\{C, D\}$ are overdemanded. Furthermore, $\left|N^{D}\left(p^{\min },\{A, B, C\}\right)\right|=$ $1<|\{A, B, C\}|=3$, and $\left|N^{D}\left(p^{\min },\{A, B, D\}\right)\right|=3 \leq|\{A, B, D\}|=3$. Similarly, $\{A, C, D\}$ nor $\{B, C, D\}$ are overdemanded. Thus, no set is ovedemanded. For the objects with positive prices, namely, $B$ and $D,\left|N^{W D}\left(p^{\min },\{B\}\right)\right|=2>|\{B\}|=1,\left|N^{W D}\left(p^{\min },\{D\}\right)\right|=$ $2>|\{D\}|=1$ and $\left|N^{W D}\left(p^{\min },\{B, D\}\right)\right|=3>|\{B, D\}|=2$. Thus, no set of is weakly underdemanded.

Fact 4 (Demange and Gale, 1985; Miyake, 1998; Morimoto and Serizawa, 2015; Alaei et al, 2016)(Demand connectedness). Let $R \in \mathcal{R}^{n}$ and $\left(z, p^{\min }(R)\right) \in W^{\min }(R)$. For each $x \in M$ with $p_{x}^{\min }(R)>0$, there is a sequence $\left\{i_{k}\right\}_{k=1}^{K}$ of $K$ distinct agents such that (i) $x_{i_{1}}=0$ or $p_{x_{i_{1}}}^{\min }(R)=0$, (ii) for each $k \in\{2, \cdots, K\}, x_{i_{k}} \neq 0$ and $p_{x_{i_{k}}}^{\min }(R)>0$, (iii) $x_{i_{K}}=x$ and (iv) for each $k \in\{1, \cdots, K-1\},\left\{x_{i_{k}}, x_{i_{k+1}}\right\} \in D\left(R_{i_{k}}, p^{\min }(R)\right)$.

Example 3. Figures 5 and 6 illustrate Fact 4. In Figure 5 , objects $B\left(=x_{2}\right)$ and $D\left(=x_{3}\right)$ are connected to object $A\left(=x_{1}\right)$ by agent 1's demand. In Figure 6 , object $D\left(=x_{3}\right)$ is connected to object $C\left(=x_{2}\right)$ by agent 2 's demand. Object $C\left(=x_{2}\right)$ is connected to object $B\left(=x_{1}\right)$ by agent 1 's demand.

\subsection{Minimum price rule}

Definition: A rule $f$ on $\mathcal{R}^{n}$ is called a minimum price (MP) rule if for each $R \in \mathcal{R}^{n}$, $f(R) \in Z^{\min }(R)$.

The following fact shows the characterization of minimum price rule on $\left(\mathcal{R}^{C}\right)^{n}$.

Fact 5 (Morimoto and Serizawa, 2015). Let $\mathcal{R} \equiv \mathcal{R}^{C}$ and $n \geq m+1$. A rule $f$ on $\mathcal{R}^{n}$ satisfies efficiency, strategy-proofness, individual rationality and no subsidy for losers if and only if it is a minimum price rule: for each $R \in \mathcal{R}^{n}, f(R) \in Z^{\min }(R)$.

\section{Characterizations of minimum price rule on the common-object- ranking and common-tiered-object domains}

First, we consider the common-object-ranking domain. We show that the minimum price rule is the only rule satisfying efficiency, strategy-proofness, individual rationality and no subsidy on the common-object-ranking domain.

Theorem 1: Let $\mathcal{R} \equiv \mathcal{R}^{R}(\pi)$. A rule $f$ on $\mathcal{R}^{n}$ satisfies efficiency, strategy-proofness, individual rationality and no subsidy if and only if it is a minimum price rule: for each $R \in \mathcal{R}^{n}, f(R) \in$ $Z^{\min }(R)$.

Next, we consider a common-tiered-object domain with respect to an indexed family of tiers $\mathcal{T}=\left\{T_{i}\right\}_{i \in K}$ with $|K|=k$ and $2 \leq k \leq m+1$. By Remark 3(ii), Theorem 1 implies the characterization of $k=m+1$. Recall that $\mu \equiv \min \{n, m+1\}$ and $l_{0} \in K$ are such that $\sum_{l=1}^{l_{0}-1}\left|T_{l}\right|<\mu \leq \sum_{l=1}^{l_{0}}\left|T_{l}\right|$.

Theorem 2: Let $\mathcal{R} \equiv \mathcal{R}^{T}(\mathcal{T})$ and $2 \leq k<m+1$. Assume that $\left|T_{l_{0}}\right|=1$. Then, a rule $f$ on $\mathcal{R}^{n}$ satisfies the axioms of Theorem 1 if and only if it is a minimum price rule: for each $R \in \mathcal{R}^{n}$, $f(R) \in Z^{\min }(R)$. 
Remark 6: (i) In Theorem 2, for the case of $k=2,\left|T_{l_{0}}\right|=1$ implies $n \geq m+1$. Then the characterization result of Theorem 2 coincides with that of Morimoto and Serizawa (2015).

(ii) In contrast to Fact 5, no additional assumption is made on the numbers of agents and objects for Theorems 1 and 2

Remark 7: In Morimoto and Serizawa (2015), efficiency, strategy-proofness, individual rationality, and no subsidy for losers implies no subsidy. In our characterizations, such an argument still holds when $n \geq m+1$. However, for the case where $n \leq m$, no subsidy is not implied by efficiency, strategy-proofness, individual rationality, and no subsidy for losers. Consider the MP rule with negative entry fee on a common-object-ranking domain. This rule satisfies efficiency, strategy-proofness, and individual rationality. ${ }^{13}$ Since $n \leq m$, no agent is a loser and such a rule satisfies no subsidy for losers. However, for the agent who receives $\pi(n)$, he receives a subsidy (the negative entry fee). A similar example can be given on a common-tiered-object domain.

The "only if" parts of Theorems 1 and 2 fail if we drop any one of the four axioms. We fix $\mathcal{R} \equiv \mathcal{R}^{R}(\pi)$ and take Theorem 1 as an example.

Example 1 (Dropping Efficiency). Let $f$ be the no-trade rule that for each preference profile, it assigns $(0,0)$ to each agent. Then, $f$ satisfies strategy-proofness, individual rationality, and no subsidy, but not efficiency.

Example 2 (Dropping Strategy-proofness). Let $f$ be the maximum equilibrium rule that for each preference profile, it selects the maximum price equilibrium. By Facts 1 and 2, for each preference profile, there is a unique maximum equilibrium price. Then, $f$ satisfies efficiency, individual rationality, and no subsidy, but not strategy-proofness.

Example 3 (Dropping Individual rationality). Let $f$ be the MP rule with positive entry fee for each agent and $n \geq m+1$. Then, $f$ satisfies efficiency, strategy-proofness, and no subsidy, but not individual rationality. ${ }^{14}$

Example 4 (Dropping No subsidy). Let $f$ be the MP rule with negative entry fee for each agent and $n \leq m$. Then, $f$ satisfies efficiency, strategy-proofness, and individual rationality, but not no subsidy. ${ }^{15}$

Similar examples can be given to show the independence of axioms for Theorem 2 .

\section{Concluding Remark}

We use efficiency, strategy-proofness, individual rationality, and no subsidy to characterize the MP rule on the common-object-ranking and common-tiered-object domains. Two open questions remain. The first is to introduce indifference to the preferences ranking objects according to $\pi$ and the preferences ranking objects according to $\mathcal{T}$. The second is to investigate the case where there may be several copies for each object. Our proofs depend on the heterogenous

\footnotetext{
${ }^{13}$ See Morimoto and Serizawa (2015).

${ }^{14} n \geq m+1$ implies that there is $i \in N$ such that $x_{i}=0$. Since $i$ pays a positive entry fee $e_{i}$, then, $(0,0) P_{i}\left(0, e_{i}\right)$, violating individual rationality.

${ }^{15}$ See Remark 7 for details.
} 
objects assumption. We believe the MP rule is still the only rule satisfying those four axioms for above-mentioned open questions.

\section{References}

[1] Adachi, T., 2014. Equity and the Vickrey allocation rule on general preference domains. Social Choice and Welfare 42(4), 813-830.

[2] Alaei, S., Kamal, J., Malekian, A., 2016. Competitive equilibrium in two-sided matching markets with general utility functions. Operations Research (Articles in Advance), 1-8.

[3] Alkan, A., Demange, G., Gale, D., 1991. Fair allocation of indivisible goods and criteria of justice. Econometrica 59(4), 1023-1039.

[4] Alkan, A., Gale, D., 1990. The core of the matching game. Games and Economic Behavior $2(3), 203-212$.

[5] Akahoshi, T., 2014. A necessary and sufficient condition for stable matching rules to be strategy-proof. Social Choice and Welfare 43(3), 683-702.

[6] Andersson, T., Ehlers, L., Svensson, L. G., 2015. Transferring ownership of public housing to existing tenants: a market design approach. Mimeo.

[7] Andersson, T., Svensson, L. G., Yang, Z., 2010. Constrainedly fair job assignments under minimum wages. Games and Economic Behavior 68(2), 428-442.

[8] Andersson, T., Svensson, L-G., 2014. Non-manipulable house allocation with rent control. Econometrica 82(2), 507-539.

[9] Baisa, B. H., 2015(a). Auction design without quasilinear preferences. Theoretical Economics, forthcoming.

[10] Baisa, B. H., 2015(b). Overbidding and inefficiencies in multi-unit Vickrey auctions for normal goods. Mimeo.

[11] Demange, G., Gale, D., 1985. The strategy structure of two-sided matching markets. Econometrica 53(4), 873-888.

[12] Heo, E. J., 2014. The extended serial correspondence on a rich preference domain. International Journal of Game Theory 43(2), 439-454.

[13] Kandori M, Kojima F, Yasuda Y, 2010. Tiers, preference similarity, and the limits on stable partners. Mimeo.

[14] Kaneko, M., 1983. Housing markets with indivisibilities. Journal of Urban Economics 13(1), $22-50$. 
[15] Kaneko, M., Ito, T., Osawa, Y. I., 2006. Duality in comparative statics in rental housing markets with indivisibilities. Journal of Urban Economics 59(1), 142-170.

[16] Kazumura, T., Serizawa, S., 2015. Efficiency and strategy-proofness in object assignment problems with multi demand preferences. ISER Discussion Paper No. 943, Osaka University.

[17] Kesten, O., 2010. School choice with consent. Quarterly Journal of Economics 125, 12971348.

[18] Kesten, O., Kurino, M., 2013. Do outside options matter in school choice? A new perspective on the efficiency vs. strategy-proofness trade-off. Mimeo.

[19] Leonard, H.B., 1983. Elicitation of honest preferences for the assignment of individuals to positions. Journal of Political Economy 91(3), 461-479.

[20] Mishra, D., Talman, D., 2010. Characterization of the Walrasian equilibria of the assignment model. Journal of Mathematical Economics 46(1), 6-20.

[21] Miyake, M., 1998. On the incentive properties of multi-item auctions. International Journal of Game Theory 27(1), 1-19.

[22] Morimoto, S., Serizawa, S., 2015. Strategy-proofness and efficiency with non-quasi-linear preferences: a characterization of minimum price Walrasian rule. Theoretical Economics $10(2), 445-487$.

[23] Morimoto, S., 2016. Strategy-proofness, efficiency, and the core in matching problems with transfers. Mimeo.

[24] Sai, S., 2016. Comparative statics in housing markets with indivisibilities: how rising income inequality affects on housing rents. Mimeo.

[25] Saitoh, H., Serizawa, S., 2008. Vickrey allocaiton rule with income effect. Economic Theory $35(2), 391-401$.

[26] Sakai, T., 2008. Second price auctions on general preference domains: two characterizations. Economic Theory 37(2), 347-356.

[27] Sun, N., Yang, Z., 2003. A general strategy proof fair allocation mechanism. Economics Letters 81(1), 73-79.

[28] Thomson, W., 2015. Strategy-proof allocation rules. Mimeo.

[29] Tierney, R., 2015. Managing multiple commons: strategy-proofness and min-price Walras. Mimeo.

[30] Vickrey, W., 1961. Counterspeculation, auctions, and competitive sealed tenders. The Journal of Finance 16(1), 8-37. 


\section{Appendix}

We owe Morimoto and Serizawa (2015) for the structure of proofs that they have developed. However, we emphasize that our domains are smaller than theirs and their proofs often employ preferences outside our domains. Thus, even in the cases where their proof techniques can be applied, we have to modify them carefully and in some cases we need to develop new proof techniques.

\section{Part A: Proof of Theorems 1}

Let $\mathcal{R} \subseteq \mathcal{R}^{R}(\pi)$. Recall that $\pi=(\pi(1), \ldots, \pi(m), \pi(m+1)), \pi(m+1)=0, \mu \equiv \min \{n, m+$ $1\}$, and for every pair $x, y \in L, x>_{\pi} y$ means that $x$ has a higher rank than $y$ according to $\pi$. Let $M_{0} \equiv\{\pi(1), \cdots, \pi(\mu)\}$ and $M_{1} \equiv\{\pi(1), \cdots, \pi(\mu-1)\}$.

Lemma A.1: Let $f$ satisfy efficiency. Let $R \in(\mathcal{R})^{n}$. Then, (a) for each $x \in M_{0}$, there is $i \in N$ such that $x_{i}(R)=x$, and (b) for each $i \in N, x_{i}(R) \in M_{0}$.

Proof: (a) By contradiction, suppose that there is $x \in M_{0}$ such that for each $i \in N$, $x_{i}(R) \neq x$. By the definition of $M_{0}$, there is $i \in N$ such that $x>_{\pi} x_{i}(R)$.

Define $z^{\prime}$ by: (i) $z_{i}^{\prime} \equiv\left(x, t_{i}(R)\right)$, and (ii) for each $j \in N \backslash\{i\}, z_{j}^{\prime} \equiv f_{j}(R)$. Then, by $R \in(\mathcal{R})^{n}$, $\left(x, t_{i}(R)\right) P_{i}\left(\pi(\mu), t_{i}(R)\right)$. Thus, $z^{\prime}$ dominates $f(R)$, contradicting efficiency.

(b) If $n \geq m+1$ (i.e., $\mu=m+1$ and $\pi(\mu)=0$ ), then $M_{0}=L$ and (b) holds trivially. If $n \leq m$ (i.e., $\mu=n$ and $\pi(\mu)>_{\pi} 0$ ), then $\left|M_{0}\right|=n$ and (b) follows from (a).

Q.E.D.

Lemma A.2: Let $f$ satisfy efficiency, strategy-proofness, and individual rationality. Let $R \in(\mathcal{R})^{n}$. Then, for each $i \in N, f_{i}(R) R_{i}(\pi(\mu), 0)$.

Proof: By contradiction, suppose that there is $i \in N$ such that $(\pi(\mu), 0) P_{i} f_{i}(R)$.

Claim: For each $x \in M_{0}, V_{i}\left(x ; f_{i}(R)\right)>0$.

By contradiction, suppose that there is $x \in M_{0}$ such that $V_{i}\left(x ; f_{i}(R)\right) \leq 0$. Then,

$$
f_{i}(R) I_{i}\left(x, V_{i}\left(x ; f_{i}(R)\right)\right) \underset{V_{i}\left(x ; f_{i}(R)\right) \leq 0}{R_{i}}(x, 0) \underset{x \in M_{0}}{R_{i}}(\pi(\mu), 0)
$$

contradicting $(\pi(\mu), 0) P_{i} f_{i}(R)$. Thus the Claim holds.

By the above Claim, there is $\widehat{R}_{i}$ such that for each $x \in M_{0}, \widehat{V}_{i}(x ; \mathbf{0})<V_{i}\left(x ; f_{i}(R)\right)$. By Lemma A.1(b), $x_{i}\left(\widehat{R}_{i}, R_{-i}\right) \in M_{0}$. Thus,

$$
t_{i}\left(\widehat{R}_{i}, R_{-i}\right) \underset{\text { individual rationality }}{\leq} \widehat{V}_{i}\left(x_{i}\left(\widehat{R}_{i}, R_{-i}\right) ; \mathbf{0}\right)<V_{i}\left(x_{i}\left(\widehat{R}_{i}, R_{-i}\right) ; f_{i}(R)\right) .
$$

Thus $f_{i}\left(\widehat{R}_{i}, R_{-i}\right) \stackrel{\text { truth }}{P_{i}} f_{i}\left(\stackrel{\text { truth }}{R_{i}}, R_{-i}\right)$, contradicting strategy-proofness.

Q.E.D.

Lemma A.3: Let $f$ satisfy the four axioms of Theorem 1. Let $R \in(\mathcal{R})^{n}$. Then, for each $i \in N$, if $x_{i}(R)=\pi(\mu), t_{i}(R)=0$.

Proof: Let $i \in N$ be such that $x_{i}(R)=\pi(\mu)$. By Lemma A.2, $f_{i}(R) R_{i}(\pi(\mu), 0)$. Thus $t_{i}(R) \leq 0$ while no subsidy implies $t_{i}(R) \geq 0$. Thus, $t_{i}(R)=0$.

Q.E.D.

Lemma A.4: Let $R \in(\mathcal{R})^{n}, i, j \in N$ and $z \in Z$ be such that $z_{i} R_{i} z_{j}$ and $z_{i} P_{j} z_{j}$. Assume that $t_{j}-V_{i}\left(x_{j} ; z_{i}\right)<V_{j}\left(x_{i} ; z_{j}\right)-t_{i}$. Then, there is $z^{\prime} \in Z$ that dominates $z$. 
Proof: Let $t_{i}^{\prime} \equiv V_{i}\left(x_{j} ; z_{i}\right)$ and $t_{j}^{\prime} \equiv t_{i}+t_{j}-V_{i}\left(x_{j} ; z_{i}\right)$.

Define $z^{\prime}$ by: (i) $z_{i}^{\prime} \equiv\left(x_{j}, t_{i}^{\prime}\right)$, (ii) $z_{j}^{\prime} \equiv\left(x_{i}, t_{j}^{\prime}\right)$, and (iii) for each $k \in N \backslash\{i, j\}, z_{k}^{\prime} \equiv z_{k}$. Then, $z_{i}^{\prime} I_{i} z_{i}$, and for each $k \in N \backslash\{i, j\}, z_{k}^{\prime} I_{k} z_{k}$. Since $t_{j}+t_{i}-V_{i}\left(x_{j} ; z_{i}\right)<V_{j}\left(x_{i} ; z_{j}\right)$, then $z_{j}^{\prime} P_{j} z_{j}$. Moreover,

$$
\sum_{k \in N} t_{k}^{\prime}=\sum_{k \in N \backslash\{i, j\}} t_{k}^{\prime}+t_{i}^{\prime}+t_{j}^{\prime}=\sum_{k \in N \backslash\{i, j\}} t_{k}^{\prime}+t_{i}+t_{j}=\sum_{k \in N} t_{k} .
$$

Thus, $z^{\prime}$ dominates $z$.

Q.E.D.

Given $z_{i} \equiv\left(x_{i}, t_{i}\right) \in L \times \mathbb{R}$ and $R_{i} \in \mathcal{R}, R_{i}^{\prime} \in \mathcal{R}$ is a semi-Maskin monotonic transformation of $R_{i}$ at $z_{i}$ if (i) for each $y<_{\pi} x_{i}, V_{i}^{\prime}\left(y ; z_{i}\right)<0$, and (ii) for each $y>_{\pi} x_{i}, V_{i}^{\prime}\left(y ; z_{i}\right)<V_{i}\left(y ; z_{i}\right)$. Let $R_{S M M}\left(R_{i}, z_{i}\right)$ be the set of semi-Maskin monotonic transformations of $R_{i}$ at $z_{i}$.

Lemma A.5: Let $f$ satisfy strategy-proofness and no subsidy. Let $R \in(\mathcal{R})^{n}$ and $R_{i}^{\prime}$ $\in R_{S M M}\left(R_{i}, f_{i}(R)\right)$. Then, $f_{i}\left(R_{i}^{\prime}, R_{-i}\right)=f_{i}\left(R_{i}, R_{-i}\right)$.

Proof: Strategy-proofness implies

$$
f_{i}\left(\stackrel{\text { truth }}{R_{i}^{\prime}}, R_{-i}\right) \stackrel{\text { truth }}{R_{i}^{\prime}} f_{i}\left(\stackrel{\text { lie }}{R_{i}}, R_{-i}\right)
$$

Thus, $\left.t_{i}\left(R_{i}^{\prime}, R_{-i}\right) \leq V_{i}^{\prime}\left(x_{i}\left(R_{i}^{\prime}, R_{-i}\right) ; f_{i}(R)\right)\right)$.

If $x_{i}\left(R_{i}^{\prime}, R_{-i}\right)<_{\pi} x_{i}(R)$, then by $R_{i}^{\prime} \in R_{S M M}\left(R_{i}, f_{i}(R)\right)$, we have:

$\left.t_{i}\left(R_{i}^{\prime}, R_{-i}\right) \leq V_{i}^{\prime}\left(x_{i}\left(R_{i}^{\prime}, R_{-i}\right) ; f_{i}(R)\right)\right)<0$, contradicting no subsidy. Thus, $x_{i}\left(R_{i}^{\prime}, R_{-i}\right) \geq_{\pi} x_{i}(R)$.

Suppose $x_{i}\left(R_{i}^{\prime}, R_{-i}\right)>_{\pi} x_{i}(R)$. Then by $\left.t_{i}\left(R_{i}^{\prime}, R_{-i}\right) \leq V_{i}^{\prime}\left(x_{i}\left(R_{i}^{\prime}, R_{-i}\right) ; f_{i}(R)\right)\right)$,

$$
f_{i}\left(R_{i}^{\prime}, R_{-i}\right) R_{i}\left(x_{i}\left(R_{i}^{\prime}, R_{-i}\right), V_{i}^{\prime}\left(x_{i}\left(R_{i}^{\prime}, R_{-i}\right) ; f_{i}(R)\right)\right) .
$$

Thus by $R_{i}^{\prime} \in R_{S M M}\left(R_{i}, f_{i}(R)\right)$,

$$
\left(x_{i}\left(R_{i}^{\prime}, R_{-i}\right), V_{i}^{\prime}\left(x_{i}\left(R_{i}^{\prime}, R_{-i}\right) ; f_{i}(R)\right)\right) P_{i}\left(x_{i}\left(R_{i}^{\prime}, R_{-i}\right), V_{i}\left(x_{i}\left(R_{i}^{\prime}, R_{-i}\right) ; f_{i}(R)\right)\right) I_{i} f_{i}(R) .
$$

Thus, $f_{i}\left(R_{i}^{\prime}, R_{-i}\right) \stackrel{\text { truth }}{P_{i}} f_{i}\left(\stackrel{\text { truth }}{R_{i}}, R_{-i}\right)$, violating strategy-proofness. Thus $x_{i}\left(R_{i}^{\prime}, R_{-i}\right)=x_{i}(R)$.

Again, by strategy-proofness, $f_{i}\left(\stackrel{\text { truth }}{R_{i}}, R_{-i}\right) \stackrel{\text { truth }}{R_{i}} f_{i}\left(\stackrel{\text { lie }}{R_{i}^{\prime}}, R_{-i}\right)$ and $f_{i}\left(\stackrel{\text { truth }}{R_{i}^{\prime}}, R_{-i}\right) \stackrel{\text { truth }}{R_{i}^{\prime}} f_{i}\left(\stackrel{\text { lie }}{R_{i}}, R_{-i}\right)$. Thus, by $x_{i}\left(R_{i}^{\prime}, R_{-i}\right)=x_{i}(R)$, we have $t_{i}\left(R_{i}^{\prime}, R_{-i}\right)=t_{i}(R)$.

Q.E.D.

Given $R \in(\mathcal{R})^{n}, x \in L$ and $z \in[L \times \mathbb{R}]^{n}$, let $\rho^{x}(R) \equiv\left(\rho_{1}^{x}(R), \cdots, \rho_{n}^{x}(R)\right)$ be the permutation on $N$ defined by $V_{\rho_{n}^{x}(R)}\left(x ; z_{\rho_{n}^{x}(R)}\right) \leq \cdots \leq V_{\rho_{1}^{x}(R)}\left(x ; z_{\rho_{1}^{x}(R)}\right)$. For each $k \in N$, let $C^{k}(R, x ; z)$ be the $k$-th highest valuation of $x$ from $z$ for $R$, i.e., $C^{k}(R, x ; z) \equiv V_{\rho_{k}^{x}(R)}\left(x ; z_{\rho_{k}^{x}(R)}\right)$.

Lemma A.6 (Morimoto and Serizawa, 2015): Let $f$ satisfy the four axioms of Theorem 1. Let $R \in(\mathcal{R})^{n}, i \in N$ and $x \equiv x_{i}(R)$. Then, $t_{i}(R) \geq C^{\mu}(R, x ;(\pi(\mu), 0))$.

Proof: By Lemma A.1(b), $x \in M_{0}$ and $x \geq_{\pi} \pi(\mu)$.

Case 1: $x=\pi(\mu)$. By Lemma A.3, $t_{i}(R)=0=C^{\mu}(R, \pi(\mu) ;(\pi(\mu), 0))$. 
Case 2: $x>_{\pi} \pi(\mu)$. By contradiction, suppose that $t_{i}(R)<C^{\mu}(R, x ;(\pi(\mu), 0))$. By $x>_{\pi}$ $\pi(\mu)$, there is $R_{i}^{\prime} \in R_{S M M}\left(R_{i}, f_{i}(R)\right)$ such that $-V_{i}^{\prime}\left(\pi(\mu) ; f_{i}(R)\right)<C^{\mu}(R, x ;(\pi(\mu), 0))-t_{i}(R)$. By Lemma A.5, $f_{i}\left(R_{i}^{\prime}, R_{-i}\right)=f_{i}\left(R_{i}, R_{-i}\right)$. Thus

$$
-V_{i}^{\prime}\left(\pi(\mu) ; f_{i}\left(R_{i}^{\prime}, R_{-i}\right)\right)<C^{\mu}(R, x ;(\pi(\mu), 0))-t_{i}\left(R_{i}^{\prime}, R_{-i}\right)
$$

By Lemmas A.1(b) and A.3, there is $j \in N \backslash\{i\}$ such that $f_{j}\left(R_{i}^{\prime}, R_{-i}\right)=(\pi(\mu), 0)$ and $V_{j}(x ;(\pi(\mu), 0)) \geq C^{\mu}(R, x ;(\pi(\mu), 0))$. Thus,

$$
t_{i}\left(R_{i}^{\prime}, R_{-i}\right)-V_{i}^{\prime}\left(\pi(\mu) ; f_{i}\left(R_{i}^{\prime}, R_{-i}\right)\right)<C^{\mu}(R, x ;(\pi(\mu), 0)) \leq V_{j}(x ;(\pi(\mu), 0)) .
$$

Define $z^{\prime}$ by:

(i) $z_{i}^{\prime} \equiv\left(\pi(\mu), V_{i}^{\prime}\left(\pi(\mu) ; f_{i}\left(R_{i}^{\prime}, R_{-i}\right)\right)\right)$,

(ii) $z_{j}^{\prime} \equiv\left(x, t_{i}\left(R_{i}^{\prime}, R_{-i}\right)-V_{i}^{\prime}\left(\pi(\mu) ; f_{i}\left(R_{i}^{\prime}, R_{-i}\right)\right)\right)$, and

(iii) for each $k \in N \backslash\{i, j\}, z_{k}^{\prime} \equiv f_{k}\left(R_{i}^{\prime}, R_{-i}\right)$.

Then, $z_{i}^{\prime} I_{i}^{\prime} f_{i}\left(R_{i}^{\prime}, R_{-i}\right)$ and $z_{j}^{\prime} P_{j} f_{j}\left(R_{i}^{\prime}, R_{-i}\right)$. Furthermore,

$V_{i}^{\prime}\left(\pi(\mu) ; f_{i}\left(R_{i}^{\prime}, R_{-i}\right)\right)+t_{i}\left(R_{i}^{\prime}, R_{-i}\right)-V_{i}^{\prime}\left(\pi(\mu) ; f_{i}\left(R_{i}^{\prime}, R_{-i}\right)\right)+\sum_{k \in N \backslash\{i, j\}} t_{k}\left(R_{i}^{\prime}, R_{-i}\right)=\sum_{k \in N} t_{k}\left(R_{i}^{\prime}, R_{-i}\right)$.

Thus, $z^{\prime}$ dominates $f\left(R_{i}^{\prime}, R_{-i}\right)$, contradicting efficiency.

Q.E.D.

Lemma A.7 (Morimoto and Serizawa, 2015): Let $f$ satisfy the four axioms of Theorem 1. Let $R \in(\mathcal{R})^{n}$ and $i \in N$ be such that $x \equiv x_{i}(R)>_{\pi} \pi(\mu)$. Then, $V_{i}(x ;(\pi(\mu), 0)) \geq$ $C^{\mu-1}(R, x ;(\pi(\mu), 0))$.

Proof: By contradiction, suppose that $V_{i}(x ;(\pi(\mu), 0))<C^{\mu-1}(R, x ;(\pi(\mu), 0))$. Then,

$$
V_{i}(x ;(\pi(\mu), 0)) \leq C^{\mu}(R, x ;(\pi(\mu), 0)) \underset{\text { Lemma A.6 }}{\leq} t_{i}(R) \underset{\text { Lemma A.2 }}{\leq} V_{i}(x ;(\pi(\mu), 0)) .
$$

Thus, $t_{i}(R)=V_{i}(x ;(\pi(\mu), 0))=C^{\mu}(R, x ;(\pi(\mu), 0))<C^{\mu-1}(R, x ;(\pi(\mu), 0))$. Thus, $V_{i}\left(\pi(\mu) ; f_{i}(R)\right)=$ 0 and

$$
\left|\left\{j \in N \backslash\{i\}: V_{j}(x ;(\pi(\mu), 0)) \geq C^{\mu-1}(R, x ;(\pi(\mu), 0))\right\}\right|=\mu-1 .
$$

By $x_{i}(R)=x>_{\pi} \pi(\mu), \mu=\min \{n, m+1\} \geq 2$, and Lemmas A.1 (b) and A.3, there is $j \in N \backslash\{i\}$ such that $f_{j}(R)=(\pi(\mu), 0)$ and $V_{j}(x ;(\pi(\mu), 0)) \geq C^{\mu-1}(R, x ;(\pi(\mu), 0))$. Thus

$$
V_{j}(x ;(\pi(\mu), 0))>C^{\mu}(R, x ;(\pi(\mu), 0))=t_{i}(R) .
$$

By $V_{i}\left(\pi(\mu) ; f_{i}(R)\right)=0$ and $t_{j}(R)=0$,

$$
t_{j}(R)-V_{i}\left(\pi(\mu) ; f_{i}(R)\right)=0<V_{j}(x ;(\pi(\mu), 0))-t_{i}(R) .
$$

By Lemma A.4, $f_{i}(R)$ is not efficient, a contradiction.

Q.E.D.

Given $R \in(\mathcal{R})^{n}$, let $Z^{\pi(\mu)}(R) \equiv\left\{z \in Z: z_{i} R_{i}(\pi(\mu), 0)\right.$ for each $\left.i \in N\right\}$. 
Lemma A.8: Let $f$ satisfy the four axioms of Theorem 1. Let $R \in(\mathcal{R})^{n}, i \in N, x \in M_{1}$ and $z \in Z^{\pi(\mu)}$. Assume that (8-i) for each $j \in N \backslash\{i\}, f_{j}(R) R_{j} z_{j}$, (8-ii) $V_{i}\left(x ;((\pi(\mu), 0))>C^{1}\left(R_{-i}, x ; z\right)\right.$,

(8-iii) there is $\varepsilon>0$ such that $V_{i}\left(x ;((\pi(\mu), 0))-C^{1}\left(R_{-i}, x ; z\right)>2 \varepsilon\right.$, and for each $y \in M_{1}$ such that $y<_{\pi} x$,

$$
V_{i}\left(y ;((\pi(\mu), 0))<\min \left\{C ^ { \mu - 1 } \left(R, y ;((\pi(\mu), 0)), V_{i}\left(x ;((\pi(\mu), 0))-C^{1}\left(R_{-i}, x ; z\right)-2 \varepsilon\right\},\right.\right.\right.
$$

and

(8-iv) for each $j \neq i$, each $t \in\left[0, V_{i}(m ; \mathbf{0})\right]$, each $t^{\prime} \in\left[0, V_{j}(m ; \mathbf{0})\right]$ and each $y>_{\pi} x$,

$$
t^{\prime}-V_{i}\left(x ;\left(y, t^{\prime}\right)\right)<V_{j}(y ;(x, t))-t .
$$

Then $x_{i}(R)=x$.

Proof: By contradiction, suppose $x_{i}(R) \neq x$. By Lemma A.1(b), there is $j \in N \backslash\{i\}$ such that $x_{j}(R)=x$.

Note

$$
t_{j}(R) \underset{(8-\mathrm{i})}{\leq} V_{j}\left(x ; z_{j}\right) \leq C^{1}\left(R_{-i}, x ; z\right) \underset{(8-\mathrm{ii})}{<} V_{i}(x ;((\pi(\mu), 0)) .
$$

Thus, there is $R_{j}^{\prime} \in R_{S M M}\left(R_{j}, f_{j}(R)\right)$ such that

(i) $-V_{j}^{\prime}\left(\left(\pi(\mu) ; f_{j}(R)\right)=V_{i}\left(x ;((\pi(\mu), 0))-C^{1}\left(R_{-i}, x ; z\right)-\varepsilon\right.\right.$,

(ii) for each $y \in M_{1}$ such that $y<_{\pi} x_{j}(R), V_{j}^{\prime}(y ;(\pi(\mu), 0))>V_{i}(x ;(\pi(\mu), 0))-C^{1}\left(R_{-i}, x ; z\right)-2 \varepsilon$, and,

(iii) for each $y>_{\pi} x_{j}(R),\left(8\right.$-iv) holds with respect to the pair $R_{i}$ and $R_{j}^{\prime}$.

By $R_{j}^{\prime} \in R_{S M M}\left(R_{j}, f_{j}(R)\right)$ and Lemma A.5, $f_{j}\left(R_{j}^{\prime}, R_{-j}\right)=f_{j}(R)$. Thus by (i),

(i') $-V_{j}^{\prime}\left(\left(\pi(\mu) ; f_{j}\left(R_{j}^{\prime}, R_{-j}\right)\right)=V_{i}\left(x ;((\pi(\mu), 0))-C^{1}\left(R_{-i}, x ; z\right)-\varepsilon\right.\right.$.

Let $y \equiv x_{i}\left(R_{j}^{\prime}, R_{-j}\right)$. By $f_{j}\left(R_{j}^{\prime}, R_{-j}\right)=f_{j}(R), y \neq x$. If $y>_{\pi} x$, then by (iii),

$$
t_{j}\left(R_{j}^{\prime}, R_{-j}\right)-V_{i}\left(x ; f_{i}\left(R_{j}^{\prime}, R_{-j}\right)\right)<V_{j}^{\prime}\left(y ; f_{j}\left(R_{j}^{\prime}, R_{-j}\right)\right)-t_{i}\left(R_{j}^{\prime}, R_{-j}\right) .
$$

By Lemma A.4, $f\left(R_{j}^{\prime}, R_{-j}\right)$ is not efficient, a contradiction. Thus, by $y \neq x, y<_{\pi} x$.

If $y \in M_{1}$, then

$$
V_{i}(y ;(\pi(\mu), 0)) \underset{(8-\mathrm{iii})}{<} V_{i}\left(x ;((\pi(\mu), 0))-C^{1}\left(R_{-i}, x ; z\right)-2 \varepsilon \underset{(\mathrm{ii})}{<} V_{j}^{\prime}(y ;(\pi(\mu), 0)) .\right.
$$

Since (8-iii) also implies $V_{i}(y ;(\pi(\mu), 0))<C^{\mu-1}(R, y ;(\pi(\mu), 0))$, then we have

$$
V_{i}(y ;(\pi(\mu), 0))<C^{\mu-1}\left(\left(R_{j}^{\prime}, R_{-j}\right), y ;(\pi(\mu), 0)\right) .
$$

By Lemma A.7, this contradicts $y \in M_{1}$. Thus, $y<_{\pi} x$ but $y \notin M_{1}$.

By Lemmas A.1(b) and $y \notin M_{1}, x_{i}\left(R_{j}^{\prime}, R_{-j}\right)=\pi(\mu)$. Thus, by Lemma A.3, $t_{i}\left(R_{j}^{\prime}, R_{-j}\right)=0$. Thus, by (i') and $t_{j}\left(R_{j}^{\prime}, R_{-j}\right)=t_{j}(R) \leq C^{1}\left(R_{-i}, x ; z\right)$,

$$
\begin{aligned}
t_{i}\left(R_{j}^{\prime}, R_{-j}\right)-V_{j}^{\prime}\left(\pi(\mu) ; f_{j}\left(R_{j}^{\prime}, R_{-j}\right)\right) & <V_{i}\left(x ;((\pi(\mu), 0))-C^{1}\left(R_{-i}, x ; z\right)\right. \\
& \leq V_{i}(x ;(\pi(\mu), 0))-t_{j}\left(R_{j}^{\prime}, R_{-j}\right) .
\end{aligned}
$$


Thus, by Lemma A.4, $f\left(R_{j}^{\prime}, R_{-j}\right)$ is not efficient, a contradiction. Thus $x_{i}(R)=x$. Q.E.D.

Proposition A.1: Let $f$ satisfy the four axioms of Theorem 1. Let $R \in(\mathcal{R})^{n}$ and $z \in$ $Z^{\min }(R)$. Then, for each $i \in N, f_{i}(R) R_{i} z_{i}$.

Proof: Without loss of generality, let $\pi=(\pi(1), \pi(2) \cdots, \pi(m+1))=(m, \cdots, 1,0)$. Let $x_{0} \equiv \max \{0, m-n+1\}$. By $\mu \equiv \min \{n, m+1\}$, we have $\mu=m-x_{0}+1$ and $\pi(\mu)=x_{0}$. If $m>n$, then $x_{0}=m-n+1$. If $m \leq n$, then $x_{0}=0$. Note $M_{0} \equiv\left\{x_{0}, \cdots, m\right\}$ and $M_{1} \equiv\left\{x_{0}+1, \cdots, m\right\}$. We only prove $f_{1}(R) R_{1} z_{1}$. For each $j \in N \backslash\{1\}, f_{j}(R) R_{j} z_{j}$ can be proved similarly.

Case $1 x_{1}=x_{0}$. By Lemma A.3, $z_{1}=\left(x_{0}, 0\right)$. By Lemma A.2, $f_{1}(R) R_{1} z_{1}$.

Case $2 x_{1}>x_{0}$. Let $N^{x_{0}} \equiv\left\{i \in N \mid x_{i}>x_{0}\right\}$. By contradiction, suppose that $z_{1} P_{1} f_{1}(R)$.

Claim 1: For each $k=0,1,2, \ldots$, there are a set $N(k+1)$ of $k+1$ distinct agents, saying $N(k+1) \equiv\{1,2, \ldots, k+1\}$, and $R_{N(k+1)}^{\prime} \in(\mathcal{R})^{k+1}$ such that:

(1-i) $z_{k+1} P_{k+1} f_{k+1}\left(R_{N(k)}^{\prime}, R_{-N(k)}\right)$;

(1-ii) $p_{x_{k+1}}^{\min }(R)<V_{k+1}^{\prime}\left(x_{k+1} ;\left(x_{0}, 0\right)\right)<V_{k+1}\left(x_{k+1} ; f_{k+1}\left(R_{N(k)}^{\prime}, R_{-N(k)}\right)\right)$;

(1-iii) for each $j \in N(k+1)$,

$\left(1\right.$-iii-a) there is $\varepsilon_{j}>0$ such that $V_{j}^{\prime}\left(x_{j} ;\left(x_{0}, 0\right)\right)-p_{x_{j}}^{\min }(R)>2 \varepsilon_{j}$, and for each $y \in M_{1}$ such that $y<x_{j}$,

$$
\begin{aligned}
& V_{j}^{\prime}\left(y ;\left(x_{0}, 0\right)\right) \\
< & \min \left\{C^{m-x_{0}+1}\left(\left(R_{\{1, \ldots j-1\}}^{\prime}, R_{N \backslash\{1, \ldots j-1\}}\right), y ;\left(x_{0}, 0\right)\right), V_{j}^{\prime}\left(x_{j} ;\left(x_{0}, 0\right)\right)-p_{x_{j}}^{\min }(R)-2 \varepsilon_{j}, V_{j}\left(y ;\left(x_{0}, 0\right)\right)\right\},
\end{aligned}
$$

(1-iii-b) for each $y>x_{j}$

(1-iii-(b-1)) for each $i \in\{1, \cdots, j-1\}$, each $t \in\left[0, V_{i}^{\prime}(m ; \mathbf{0})\right]$ and each $t^{\prime} \in\left[0, V_{j}^{\prime}(m ; \mathbf{0})\right]$,

$$
t^{\prime}-V_{j}^{\prime}\left(x_{j} ;\left(y, t^{\prime}\right)\right)<V_{i}^{\prime}\left(y ;\left(x_{j}, t\right)\right)-t
$$

(1-iii-(b-2)) for each $i \in\{j+1, \cdots, n\}$, each $t \in\left[0, V_{i}(m ; \mathbf{0})\right]$ and each $t^{\prime} \in\left[0, V_{j}^{\prime}(m ; \mathbf{0})\right]$,

$$
t^{\prime}-V_{j}^{\prime}\left(x_{j} ;\left(y, t^{\prime}\right)\right)<V_{i}\left(y ;\left(x_{j}, t\right)\right)-t
$$

and

$(1-\mathrm{iii}-(\mathrm{b}-3)) V_{j}^{\prime}\left(y ;\left(x_{0}, 0\right)\right)<p_{y}^{\min }(R) ;$

(1-iv) $N(k+1) \varsubsetneqq N^{x_{0}}$.

We inductively prove Claim 1.

Step 1: We prove Claim 1 for the case of $k=0$.

Note $N(1)=\{1\}$. By $z_{1} P_{1} f_{1}(R),(1-\mathrm{i}-1)$ holds and $p_{x_{1}}^{\min }(R)<V_{1}\left(x_{1} ; f_{1}(R)\right)$. Thus, there is $R_{1}^{\prime} \in \mathcal{R}$ such that

$(1-\mathrm{ii}-1) p_{x_{1}}^{\min }(R)<V_{1}^{\prime}\left(x_{1} ;\left(x_{0}, 0\right)\right)<V_{1}\left(x_{1} ; f_{1}(R)\right)$;

(1-iii-a-1) there is $\varepsilon_{1}>0$ such that $V_{1}^{\prime}\left(x_{1} ;((\pi(\mu), 0))-p_{x_{1}}^{\min }(R)>2 \varepsilon\right.$, and for each $y \in M_{1}$ such that $y<x_{1}$,

$$
V_{1}^{\prime}\left(y ;\left(x_{0}, 0\right)\right)<\min \left\{C^{m-x_{0}+1}\left(R, y ;\left(x_{0}, 0\right)\right), V_{1}^{\prime}\left(x_{1} ;((\pi(\mu), 0))-p_{x_{1}}^{\min }(R)-2 \varepsilon_{1}, V_{1}\left(y ;\left(x_{0}, 0\right)\right)\right\}\right.
$$


(1-iii-b-1) for each $y>x_{1}$

(1-iii-(b-2)-1) for each $i \in N \backslash\{1\}$, each $t \in\left[0, V_{i}(m ; \mathbf{0})\right]$ and each $t^{\prime} \in\left[0, V_{1}^{\prime}(m ; \mathbf{0})\right]$,

$$
t^{\prime}-V_{1}^{\prime}\left(x_{1} ;\left(y, t^{\prime}\right)\right)<V_{i}\left(y ;\left(x_{1}, t\right)\right)-t,
$$

and

(1-iii-(b-3)-1) $V_{1}^{\prime}\left(y ;\left(x_{0}, 0\right)\right)<p_{y}^{\min }(R){ }^{16}$

Then, by the construction of $R_{1}^{\prime},(1-i i-1)$ and (1-iii-1) holds. Thus, we prove (1-iv-1), i.e., $N(1) \varsubsetneqq N^{x_{0}}$. By Lemma A.2, $z_{1} P_{1} f_{1}(R) R_{1}\left(x_{0}, 0\right)$. Thus $1 \in N^{x_{0}}$. Thus, $N(1)=\{1\} \subseteq N^{x_{0}}$. By contradiction, suppose that $N^{x_{0}}=\{1\}$. Then by Lemma A.1, $\mu=\min \{n, m+1\}=2$, which implies $n=2$ or $m=1$. Thus, by Lemma A.1, for each $j \in N \backslash\{1\}, z_{j}=\left(x_{0}, 0\right)$.

By $z \in Z^{\min }(R), z \in Z^{x_{0}}$. We show (8-i), (8-ii), (8-iii) and (8-iv) of Lemma A.8 with respect to $z$ to conclude $x_{1}\left(R_{1}^{\prime}, R_{N \backslash\{1\}}\right)=x_{1}$.

By Lemma A.2, for each $j \in N \backslash\{1\}, f_{j}\left(R_{1}^{\prime}, R_{N \backslash\{1\}}\right) R_{j}\left(x_{0}, 0\right)=z_{j}$. Thus, (8-i) holds. (1-iii-b-1) implies (8-iv).

Note that for each $j \in N \backslash\{1\}$, by $z \in Z^{\min }(R),\left(x_{0}, 0\right)=z_{j} R_{j} z_{1}$ and $V_{j}\left(x_{1} ;\left(x_{0}, 0\right)\right) \leq$ $p_{x_{1}}^{\min }(R)$. Thus $C^{1}\left(R_{-i}, x_{1} ; z\right) \leq p_{x_{1}}^{\min }(R)$, and so by (1-ii-1), (8-ii) holds. By $C^{1}\left(R_{-i}, x_{1} ; z\right) \leq$ $p_{x_{1}}^{\min }(R)$ and (1-iii-a-1),

$$
0<V_{1}^{\prime}\left(x_{1} ;\left(x_{0}, 0\right)\right)-p_{x_{1}}^{\min }(R)-2 \varepsilon_{1} \leq V_{1}^{\prime}\left(x_{1} ;\left(x_{0}, 0\right)\right)-C^{1}\left(R_{-i}, x_{1} ; z\right)-2 \varepsilon_{1},
$$

which implies (8-iii).

Since (8-i), (8-ii), (8-iii) and (8-iv) of Lemma A.8 hold, $x_{1}\left(R_{1}^{\prime}, R_{N \backslash\{1\}}\right)=x_{1}$.

Note

$$
t_{1}\left(R_{1}^{\prime}, R_{N \backslash\{1\}}\right) \underset{\text { Lemma A.2 }}{\leq} V_{1}^{\prime}\left(x_{1} ;\left(x_{0}, 0\right)\right) \underset{(1-\mathrm{ii}-1)}{<} V_{1}\left(x_{1} ; f_{1}(R)\right) .
$$

Thus, by $x_{1}\left(R_{1}^{\prime}, R_{N \backslash\{1\}}\right)=x_{1}$,

$$
\left.f_{1}\left(\stackrel{\text { lie }}{R_{1}^{\prime}}, R_{N \backslash\{1\}}\right) \stackrel{\text { truth }}{P_{1}} f_{1} \stackrel{\text { truth }}{R_{1}}, R_{N \backslash\{1\}}\right) .
$$

This contradicts strategy-proofness. Thus, $N(1) \varsubsetneqq N^{x_{0}}$. Thus (1-iv-1) holds.

Induction hypothesis: There are a set $N(k)$ of $k>0$ distinct agents, saying $N(k)=$ $\{1,2, \ldots, k\}$, and $R_{N(k)}^{\prime} \in(\mathcal{R})^{k}$ such that:

$(1-\mathrm{i}-k) z_{k} P_{k} f_{k}\left(R_{N(k-1)}^{\prime}, R_{-N(k-1)}\right)$;

$(1-$ ii- $k) p_{x_{k}}^{\min }(R)<V_{k}^{\prime}\left(x_{k} ;\left(x_{0}, 0\right)\right)<V_{k}\left(x_{k} ; f_{k}\left(R_{N(k-1)}^{\prime}, R_{-N(k-1)}\right)\right)$;

(1-iii- $k$ ) for each $j \in N(k)$,

$(1$-iii-a- $k)$ there is $\varepsilon_{j}>0$ such that $V_{j}^{\prime}\left(x_{j} ;\left(x_{0}, 0\right)\right)-p_{x_{j}}^{\min }(R)>2 \varepsilon_{j}$, and for each $y \in M_{1}$ such that $y<x_{j}$,

$$
\begin{aligned}
& V_{j}^{\prime}\left(y ;\left(x_{0}, 0\right)\right) \\
< & \min \left\{C^{m-x_{0}+1}\left(\left(R_{\{1, \ldots, j-1\}}^{\prime}, R_{N \backslash\{1, \ldots, j-1\}}\right), y ;\left(x_{0}, 0\right)\right), V_{j}^{\prime}\left(x_{j} ;\left(x_{0}, 0\right)\right)-p_{x_{j}}^{\min }(R)-2 \varepsilon_{j}, V_{j}\left(x_{j} ;\left(x_{0}, 0\right)\right)\right\},
\end{aligned}
$$

\footnotetext{
${ }^{16}(1-$ iii-(b-1)-1) is satisfied vacuously.
} 
(1-iii-b- $k$ ) for each $y>x_{j}$

(1-iii-(b-1)-k) for each $i \in\{1, \cdots, j-1\}$, each $t \in\left[0, V_{i}^{\prime}(m ; \mathbf{0})\right]$ and each $t^{\prime} \in\left[0, V_{j}^{\prime}(m ; \mathbf{0})\right]$,

$$
t^{\prime}-V_{j}^{\prime}\left(x_{j} ;\left(y, t^{\prime}\right)\right)<V_{i}^{\prime}\left(y ;\left(x_{j}, t\right)\right)-t,
$$

(1-iii-(b-2)- $k$ ) for each $i \in\{j+1, \cdots, n\}$, each $t \in\left[0, V_{i}(m ; \mathbf{0})\right]$ and each $t^{\prime} \in\left[0, V_{j}^{\prime}(m ; \mathbf{0})\right]$,

$$
t^{\prime}-V_{j}^{\prime}\left(x_{j} ;\left(y, t^{\prime}\right)\right)<V_{i}\left(y ;\left(x_{j}, t\right)\right)-t
$$

and

$(1-$ iii- $(\mathrm{b}-3)-k) V_{j}^{\prime}\left(y ;\left(x_{0}, 0\right)\right)<p_{y}^{\min }(R)$; $(1$-iv- $k) N(k) \varsubsetneqq N^{x_{0}}$.

Step 2: We prove Claim 1 for the case of $k+1$.

Step 2-1: We prove that there is $i \in N^{x_{0}} \backslash N(k)$ such that $z_{i} P_{i} f_{i}\left(R_{N(k)}^{\prime}, R_{-N(k)}\right)$.

By $(1$-iv- $k), N^{x_{0}} \backslash N(k) \neq \varnothing$. By contradiction, suppose that for each $i \in N^{x_{0}} \backslash N(k)$, $f_{i}\left(R_{N(k)}^{\prime}, R_{-N(k)}\right) R_{i} z_{i}$.

Let $z^{\prime}$ be such that for each $i \in N \backslash N(k), z_{i}^{\prime} \equiv z_{i}$ and for each $i \in N(k) \backslash\{k\}, z_{i}^{\prime} \equiv\left(x_{0}, 0\right)$. Then $z^{\prime} \in Z^{x_{0}}$. We show (8-i), (8-ii), (8-iii) and (8-iv) of Lemma A.8 with respect to $z^{\prime}$ to conclude $x_{k}\left(R_{N(k)}^{\prime}, R_{-N(k)}\right)=x_{k}$.

For each $i \in N \backslash N^{x_{0}}$, by $z_{i}=\left(x_{0}, 0\right)$ and Lemma A.2, $f_{i}\left(R_{N(k)}^{\prime}, R_{-N(k)}\right) R_{i} z_{i}=z_{i}^{\prime}$. For each $i \in N^{x_{0}} \backslash N(k)$, by $z_{i}^{\prime}=z_{i}, f_{i}\left(R_{N(k)}^{\prime}, R_{-N(k)}\right) R_{i} z_{i}^{\prime}$. For each $i \in N(k) \backslash\{k\}$, by Lemma A.2, $f_{i}\left(R_{N(k)}^{\prime}, R_{-N(k)}\right) R_{i}^{\prime}\left(x_{0}, 0\right)=z_{i}^{\prime}$. Thus, (8-i) holds. (1-iii-(b-1)- $k$ ) and (1-iii-(b-2)-k) imply (8-iv).

In the following, we show:

$$
C^{1}\left(\left(R_{N(k) \backslash\{k\}}^{\prime}, R_{-N(k)}\right), x_{k} ; z^{\prime}\right) \leq p_{x_{k}}^{\min }(R) .
$$

For each $i \in N \backslash N(k)$, by $z \in Z^{\min }(R), z_{i}^{\prime}=z_{i} R_{i} z_{k}$, and so $V_{i}\left(x_{k} ; z_{i}^{\prime}\right) \leq p_{x_{k}}^{\min }(R)$. For each $i \in N(k) \backslash\{k\}$, if $x_{i}>x_{k},(1$-iii-a- $k)$ implies:

$$
\begin{aligned}
V_{i}^{\prime}\left(x_{k} ; z_{i}^{\prime}\right) & =V_{i}^{\prime}\left(x_{k} ;\left(x_{0}, 0\right)\right) \\
& <C^{m-x_{0}+1}\left(\left(R_{\{1, . . i-1\}}^{\prime}, R_{N \backslash\{1, . . i-1\}}\right), x_{k} ;\left(x_{0}, 0\right)\right) \\
& \leq C^{m-x_{0}+1}\left(R, x_{k} ;\left(x_{0}, 0\right)\right) \leq p_{x_{k}}^{\min }(R)
\end{aligned}
$$

and if $x_{i}<x_{k},\left(1\right.$-iii-(b-3)-k) implies $V_{i}^{\prime}\left(x_{k} ; z_{i}^{\prime}\right)=V_{i}^{\prime}\left(x_{k} ;\left(x_{0}, 0\right)\right) \leq p_{x_{k}}^{\min }(R)$. Thus, $(*)$ holds.

By $(1$-ii- $k)$ and $(*),(8$-ii) holds.

By $(*)$ and $(1-$ iii-a- $k)$,

$$
0<V_{k}^{\prime}\left(x_{k} ;\left(x_{0}, 0\right)\right)-p_{x_{k}}^{\min }(R)-2 \varepsilon_{k} \leq V_{k}^{\prime}\left(x_{k} ;\left(x_{0}, 0\right)\right)-C^{1}\left(\left(R_{N(k) \backslash\{k\}}^{\prime}, R_{-N(k)}\right), x_{1} ; z^{\prime}\right)-2 \varepsilon_{k} .
$$

Thus, by (1-iii-a- $k)$, (8-iii) holds.

Since (8-i), (8-ii), (8-iii) and (8-iv) of Lemma A.8 hold, $x_{k}\left(R_{N(k)}^{\prime}, R_{-N(k)}\right)=x_{k}$. 
Note

$$
t_{k}\left(R_{N(k)}^{\prime}, R_{-N(k)}\right) \underset{\text { Lemma A.2 }}{\leq} V_{k}^{\prime}\left(x_{k} ;\left(x_{0}, 0\right)\right) \underset{(1-\mathrm{ii}-k)}{<} V_{k}\left(x_{k} ; f_{k}\left(R_{N(k) \backslash\{k\}}^{\prime}, R_{-((N(k) \backslash\{k\})}\right)\right) .
$$

Thus, by $x_{k}\left(R_{N(k)}^{\prime}, R_{-N(k)}\right)=x_{k}$,

$$
\left.f_{k}\left(R_{k}^{\prime}, R_{N(k) \backslash\{k\}}^{\prime}, R_{-N(k)}\right) \stackrel{\text { truth }}{P_{k}} f_{k} \stackrel{\text { truth }}{R_{k}}, R_{N(k) \backslash\{k\}}^{\prime}, R_{-N(k)}\right) .
$$

This contradicts strategy-proofness. Thus, there is $i \in N^{x_{0}} \backslash N(k)$ such that $z_{i} P_{i} f_{i}\left(R_{N(k)}^{\prime}, R_{-N(k)}\right)$.

Let $N(k+1) \equiv N(k) \cup\{i\}$. Without loss of generality, let $i \equiv k+1$. By (1-iv- $k$ ), $N(k+1) \subseteq N^{x_{0}}$. $z_{k+1} P_{k+1} f_{k+1}\left(R_{N(k)}^{\prime}, R_{-N(k)}\right)$ implies that there is $R_{k+1}^{\prime} \in \mathcal{R}$ satisfying(1-i$(k+1)),(1-\mathrm{ii}-(k+1))$, and $(1-\mathrm{iii}-(k+1))$.

Step 2-2: We prove $(1-\mathrm{iv}-(k+1))$, i.e., $N(k+1) \varsubsetneqq N^{x_{0}}$.

By contradiction, suppose that $N(k+1)=N^{x_{0}}$.

Let $z^{\prime} \in Z^{x_{0}}$ be such that for each $i \in N \backslash\{k+1\}, z_{i}^{\prime} \equiv\left(x_{0}, 0\right)$. We show (8-i), (8-ii), (8-iii) and (8-iv) of Lemma A.8 with respect to $z^{\prime}$ to conclude $x_{k+1}\left(R_{N(k+1)}^{\prime}, R_{-N(k+1)}\right)=x_{k+1}$.

By Lemma A.2, for each $i \in N \backslash N(k+1), f_{i}\left(R_{N(k+1)}^{\prime}, R_{-N(k+1)}\right) R_{i} z_{i}^{\prime}$. By Lemma A.2 again, for each $i \in N(k+1) \backslash\{k+1\}, f_{i}\left(R_{N(k)}^{\prime}, R_{-N(k)}\right) R_{i}^{\prime} z_{i}^{\prime}$. Thus, (8-i) holds. (1-iii- $\left.(\mathrm{b}-1)-(k+1)\right)$ and (1-iii-(b-2)- $(k+1))$ imply (8-iv).

In the following, we show:

$$
C^{1}\left(\left(R_{N(k+1) \backslash\{k+1\}}^{\prime}, R_{-N(k+1)}\right), x_{k+1} ; z^{\prime}\right) \leq p_{x_{k+1}}^{\min }(R) .
$$

For each $i \in N \backslash N(k+1)$, by $N(k+1)=N^{x_{0}}$, we have $z_{i}=\left(x_{0}, 0\right)$. Then, by $z \in Z^{\min }(R)$, $z_{i}^{\prime}=z_{i} R_{i} z_{k+1}$. Thus $V_{i}\left(x_{k+1} ; z_{i}^{\prime}\right) \leq p_{x_{k+1}}^{\min }(R)$. For each $i \in N(k+1) \backslash\{k+1\}$, if $x_{i}>x_{k+1}$, (1-iii-a- $(k+1))$ implies:

$$
\begin{aligned}
V_{i}^{\prime}\left(x_{k+1} ; z_{i}^{\prime}\right) & <C^{m-x_{0}+1}\left(\left(R_{\{1,2,3, . . i-1\}}^{\prime}, R_{N \backslash\{1,2,3, . . i-1\}}\right), x_{k+1} ;\left(x_{0}, 0\right)\right) \\
& \leq C^{m-x_{0}+1}\left(R, x_{k+1} ;\left(x_{0}, 0\right)\right) \leq p_{x_{k+1}}^{\min }(R)
\end{aligned}
$$

and if $x_{i}<x_{k+1},(1$-iii-(b-3)- $(k+1))$ implies $V_{i}^{\prime}\left(x_{k+1} ; z_{i}^{\prime}\right)=V_{i}^{\prime}\left(x_{k+1} ;\left(x_{0}, 0\right)\right) \leq p_{x_{k+1}}^{\min }(R)$. Thus, $(* *)$ holds.

By $(1-\mathrm{ii}-(k+1))$ and $(* *),(8-\mathrm{ii})$ holds.

By $(* *)$ and $(1-$ iii-a- $(k+1))$,

$$
\begin{aligned}
0< & V_{k+1}^{\prime}\left(x_{k+1} ;\left(x_{0}, 0\right)\right)-p_{x_{k+1}}^{\min }(R)-2 \varepsilon_{k+1} \leq \\
& V_{k+1}^{\prime}\left(x_{k+1} ;\left(x_{0}, 0\right)\right)-C^{1}\left(\left(R_{N(k+1) \backslash\{k+1\}}^{\prime}, R_{-N(k+1)}\right), x_{k+1} ; z^{\prime}\right)-2 \varepsilon_{k+1} .
\end{aligned}
$$

Thus, by (1-iii-a- $(k+1))$, (8-iii) holds.

Since (8-i), (8-ii), (8-iii) and (8-iv) of Lemma A.8 hold, $x_{k+1}\left(R_{N(k+1)}^{\prime}, R_{-N(k+1)}\right)=x_{k+1}$. 
Note

$$
\begin{aligned}
& t_{k+1}\left(R_{N(k+1)}^{\prime}, R_{-N(k+1)}\right) \underset{\text { Lemma A.2 }}{\leq} V_{k+1}^{\prime}\left(x_{k+1} ;\left(x_{0}, 0\right)\right) \\
& \underset{(1-\mathrm{ii}-(k+1))}{<} V_{k+1}\left(x_{k+1} ; f_{k+1}\left(R_{N(k)}^{\prime}, R_{-(N(k)}\right)\right) .
\end{aligned}
$$

Thus, by $x_{k+1}\left(R_{N(k+1)}^{\prime}, R_{-N(k+1)}\right)=x_{k+1}$,

$$
\left.\stackrel{\text { lie }}{f_{k+1}\left(R_{k+1}^{\prime}\right.}, R_{N(k+1) \backslash\{k+1\}}^{\prime}, R_{-N(k+1)}\right) \stackrel{\text { truth }}{P_{k+1}} f_{k+1}\left(\stackrel{\text { truth }}{R_{k+1}}, R_{N(k+1) \backslash\{k+1\}}^{\prime}, R_{-N(k)}\right) .
$$

This contradicts strategy-proofness. Thus, $(1-\mathrm{iv}-(k+1))$ holds.

By Claim 1, for each $k \geq 0, N(k+1) \varsubsetneqq N^{x_{0}}$. Let $k=m-x_{0}$. Then, $|N(k+1)|=k+1>$ $m-x_{0}=\left|N^{x_{0}}\right|$, a contradiction.

Q.E.D.

The rest of the proof of Theorem 1 is similar to the proofs of Proposition 3 and the completion of the proof of Theorem 2 in Morimoto and Serizawa (2015). Thus, we omit it.Q.E.D.

\section{Part B: Proof of Theorem 2}

Recall that $\mu \equiv \min \{n, m+1\}$ and $k$ denotes the number of object tiers. Let $2 \leq k<m+1$. Recall that $l_{0} \in K$ and $\sum_{l=1}^{l_{0}-1}\left|T_{l}\right|<\mu \leq \sum_{l=1}^{l_{0}}\left|T_{l}\right|$. Assume $\left|T_{l_{0}}\right|=1$. Then, $T_{l_{0}} \equiv\{\tau(\mu)\}$. Let $M_{0} \equiv \underset{j \in\left\{1, \cdots, l_{0}\right\}}{\cup} T_{j}$ and $M_{1} \equiv \underset{j \in\left\{1, \cdots, l_{0}-1\right\}}{\cup} T_{j}$.

Lemma B.1: Let $f$ satisfy efficiency. Let $R \in\left(\mathcal{R}^{T}(\mathcal{T})\right)^{n}$. Then, (a) for each $x \in M_{0}$, there is $i \in N$ such that $x_{i}(R)=x$, and (b) for each $i \in N, x_{i}(R) \in M_{0}$.

Proof: (a) By contradiction, suppose that there is $x \in M_{0}$ such that for each $i \in N$, $x_{i}(R) \neq x$. Since $\left|T_{l_{0}}\right|=1$, by the definitions of $l_{0}$ and $M_{0}$, there is $i \in N$ such that $x_{i}(R) \in$ $\underset{j \in\left\{l_{0}+1, \cdots, k\right\}}{\cup} T_{j}$.

Define $z^{\prime}$ by $(\mathrm{i}) z_{i}^{\prime} \equiv\left(x, t_{i}(R)\right)$, and (ii) for each $j \in N \backslash\{i\}, z_{j}^{\prime} \equiv f_{j}(R)$. Then, by $R \in$ $\left(\mathcal{R}^{T}(\mathcal{T})\right)^{n},\left(x, t_{i}(R)\right) R_{i}\left(\tau(\mu), t_{i}(R)\right) P_{i}\left(x_{i}(R), t_{i}(R)\right)$. Thus, $z^{\prime}$ dominates $f(R)$, contradicting efficiency.

(b) If $n \geq m+1, \mu=m+1$ and $\tau(\mu)=0$. Then $M_{0}=L$ and (b) holds trivially. If $n \leq m$, $\mu=n$. Then, since $\left|T_{l_{0}}\right|=1$, by the definition of $l_{0},\left|M_{0}\right|=n$ and (b) follows from (a). Q.E.D.

The proofs of Lemmas B.2 to B.7 are similar to those of Lemmas A.2 to A.7. Thus, we omit them.

Lemma B.2: Let $f$ satisfy the four axioms of Theorem 1. Let $R \in\left(\mathcal{R}^{T}(\mathcal{T})\right)^{n}$. Then, for each $i \in N, f_{i}(R) R_{i}(\tau(\mu), 0)$.

Lemma B.3: Let $f$ satisfy the four axioms of Theorem 1. Let $R \in\left(\mathcal{R}^{T}(\mathcal{T})\right)^{n}$. Then, for each $i \in N$, if $x_{i}(R)=\tau(\mu), t_{i}(R)=0$.

Lemma B.4: Let $R \in\left(\mathcal{R}^{T}(\mathcal{T})\right), i, j \in N$ and $z \in Z$ be such that $z_{i} R_{i} z_{j}$ and $z_{i} P_{j} z_{j}$. Assume that $t_{j}-V_{i}\left(x_{j} ; z_{i}\right)<V_{j}\left(x_{i} ; z_{j}\right)-t_{i}$. Then, there is $z^{\prime} \in Z$ that dominates $z$.

For each $x \in L$, let $l(x) \in K$ be such that $x \in T_{l(x)}$. Given $z_{i} \equiv\left(x_{i}, t_{i}\right) \in L \times \mathbb{R}$ and $R_{i} \in \mathcal{R}^{T}(\mathcal{T})$, a preference $R_{i} \in \mathcal{R}^{T}(\mathcal{T})$ is a semi-Maskin monotonic transformation of $R_{i}$ at 
$z_{i}$ if (i) for each $y \in \underset{j \in\{l(x), \cdots, k\}}{\cup} T_{j} \backslash\left\{x_{i}\right\}, V_{i}^{\prime}\left(y ; z_{i}\right)<0$, and (ii) for each $y \in \underset{j \in\left\{1, \cdots, l\left(x_{i}\right)-1\right\}}{\cup} T_{j}$, $V_{i}^{\prime}\left(y ; z_{i}\right)<V_{i}\left(y ; z_{i}\right)$. Let $R_{S M M}\left(R_{i}, z_{i}\right)$ be the set of semi-Maskin monotonic transformations of $R_{i}$ at $z_{i}$.

Lemma B.5: Let $f$ satisfy strategy-proofness and no subsidy. Let $R \in\left(\mathcal{R}^{T}(\mathcal{T})\right)^{n}$. Let $R_{i}^{\prime}$ $\in R_{S M M}\left(R_{i}, f_{i}(R)\right)$. Then, $f_{i}\left(R_{i}^{\prime}, R_{-i}\right)=f_{i}\left(R_{i}, R_{-i}\right)$.

Lemma B.6: Let $f$ satisfy the four axioms of Theorem 1. Let $R \in\left(\mathcal{R}^{T}(\mathcal{T})\right)^{n}$. Let $i \in N$ and $x \equiv x_{i}(R)$. Then, $t_{i}(R) \geq C^{\mu}(R, x ;(\tau(\mu), 0))$.

Lemma B.7: Let $f$ satisfy the four axioms of Theorem 1. Let $R \in\left(\mathcal{R}^{T}(\mathcal{T})\right)^{n}$. Let $i \in N$ be such that $x \equiv x_{i}(R) \in M_{1}$. Then, $V_{i}(x ;(\tau(\mu), 0)) \geq C^{\mu-1}(R, x ;(\tau(\mu), 0))$.

Given $R \in\left(\mathcal{R}^{T}(\mathcal{T})\right)^{n}$, let $Z^{\tau(\mu)} \equiv\left\{z \in Z: z_{i} R_{i}(\tau(\mu), 0)\right.$ for each $\left.i \in N\right\}$.

Lemma B.8: Let $f$ satisfy the four axioms of Theorem 1 . Let $R \in\left(\mathcal{R}^{T}(\mathcal{T})\right)^{n}$. Let $i \in N$, $x \in M_{1}$ and $z \in Z^{\tau(\mu)}$. Assume that

(8-i) for each $j \in N \backslash\{i\}, f_{j}(R) R_{j} z_{j}$,

(8-ii) $V_{i}(x ;(\tau(\mu), 0))>C^{1}\left(R_{-i}, x ; z\right)$,

(8-iii) there is $\varepsilon>0$ such that $V_{i}(x ;(\tau(\mu), 0))-C^{1}\left(R_{-i}, x ; z\right)-2 \varepsilon>0$ and for each $y \in$ $\underset{j \in\left\{l(x), \cdots, l_{0}-1\right\}}{\cup} T_{j} \backslash\{x\}$,

$$
V_{i}(y ;(\tau(\mu), 0))<\min \left\{C^{\mu-1}(R, y ;(\tau(\mu), 0)), V_{i}(x ;(\tau(\mu), 0))-C^{1}\left(R_{-i}, x ; z\right)-2 \varepsilon\right\},
$$

and

(8-iv) for each $j \neq i$, each $t \in\left[0, \max _{y \in T_{1}} V_{i}(y ; \mathbf{0})\right]$, each $t^{\prime} \in\left[0, \max _{y \in T_{1}} V_{j}(y ; \mathbf{0})\right]$ and each $y \in$ $\underset{j \in\{1, \cdots, l(x)-1\}}{\cup} T_{j}, t^{\prime}-V_{i}\left(x ;\left(y, t^{\prime}\right)\right)<V_{j}(y ;(x, t))-t$.

Then $x_{i}(R)=x$.

Proof: By contradiction, suppose $x_{i}(R) \neq x$. By Lemma B.1(b), there is $j \in N$ such that $x_{j}(R)=x$.

Note

$$
t_{j}(R) \underset{(8-\mathrm{i})}{\leq} V_{j}\left(x ; z_{j}\right) \leq C^{1}\left(R_{-i}, x ; z\right) \underset{(8-\mathrm{ii})}{<} V_{i}(x ;(\tau(\mu), 0)) .
$$

Thus, there is $R_{j}^{\prime} \in \mathcal{R}_{S M P}\left(R_{j}, f_{j}(R)\right)$ such that

(i) $-V_{j}^{\prime}\left(\tau(\mu) ; f_{j}(R)\right)=V_{i}(x ;(\tau(\mu), 0))-C^{1}\left(R_{-i}, x ; z\right)-\varepsilon$,

(ii) for each $y \in \underset{j \in\left\{l(x), \cdots, l_{0}-1\right\}}{\cup} T_{j} \backslash\{x\}, V_{j}^{\prime}(y ;(\tau(\mu), 0))>V_{i}(x ;(\tau(\mu), 0))-C^{1}\left(R_{-i}, x ; z\right)-2 \varepsilon$, and,

(iii) for each $y \in \underset{j \in\{1, \cdots, l(x)-1\}}{\cup} T_{j}$, (8-iv) holds with respect to the pair $R_{i}$ and $R_{j}^{\prime}$.

By $R_{j}^{\prime} \in \mathcal{R}_{S M P}\left(R_{j}, f_{j}(R)\right)$ and Lemma B.5, $f_{j}\left(R_{j}^{\prime}, R_{-j}\right)=f_{j}(R)$. Thus, by (i), (i') $-V_{j}^{\prime}\left(\tau(\mu) ; f_{j}\left(R_{j}^{\prime}, R_{-j}\right)\right)=V_{i}(x ;(\tau(\mu), 0))-C^{1}\left(R_{-i}, x ; z\right)-\varepsilon$.

Let $y \equiv x_{i}\left(R_{j}^{\prime}, R_{-j}\right)$. By $f_{j}\left(R_{j}^{\prime}, R_{-j}\right)=f_{j}(R), y \neq x$. If $y \in \underset{j \in\{1, \cdots, l(x)-1\}}{\cup} T_{j}$, then by (iii),

$$
t_{j}\left(R_{j}^{\prime}, R_{-j}\right)-V_{i}\left(x ; f_{i}\left(R_{j}^{\prime}, R_{-j}\right)\right)<V_{j}^{\prime}\left(y ; f_{j}\left(R_{j}^{\prime}, R_{-j}\right)\right)-t_{i}\left(R_{j}^{\prime}, R_{-j}\right) .
$$

By Lemma 5.4, $f\left(R_{j}^{\prime}, R_{-j}\right)$ is not efficient, a contradiction. Thus, by $y \neq x, y \in \underset{j \in\{l(x), \cdots, k\}}{\cup} T_{j} \backslash\{x\}$. 
If $y \in \underset{j \in\left\{l(x), \cdots, l_{0}-1\right\}}{\cup} T_{j} \backslash\{x\}$, then

$$
V_{i}(y ;(\tau(\mu), 0)) \underset{(8 \text {-iii) }}{<} V_{i}(x ;(\pi(\mu), 0))-C^{1}\left(R_{-i}, x ; z\right)-2 \varepsilon \underset{(8-\mathrm{ii})}{<} V_{j}^{\prime}(y ;(\tau(\mu), 0)) .
$$

Since (8-iii) also implies $V_{i}(y ;(\tau(\mu), 0))<C^{\mu-1}(R, y ;(\tau(\mu), 0))$, then we have

$$
V_{i}(y ;(\tau(\mu), 0))<C^{\mu-1}\left(\left(R_{j}^{\prime}, R_{-j}\right), y ;(\tau(\mu), 0)\right) .
$$

By Lemma B.7, this contradicts $y \in \underset{j \in\left\{l(x), \cdots, l_{0}-1\right\}}{\cup} T_{j} \backslash\{x\}$. Thus $y \in \underset{j \in\{l(x), \cdots, k\}}{\cup} T_{j} \backslash\{x\}$ but $y \notin \underset{i \in\left\{l(x), \cdots, l_{0}-1\right\}}{\cup} T_{i} \backslash\{x\}$.

By Lemma B.1(b) and $y \notin \underset{i \in\left\{l(x), \cdots, l_{0}-1\right\}}{\cup} T_{i} \backslash\{x\}, x_{i}\left(R_{j}^{\prime}, R_{-j}\right)=\tau(\mu)$. Thus, by Lemma A.3, $t_{i}\left(R_{j}^{\prime}, R_{-j}\right)=0$. Thus, by (i') and $t_{j}\left(R_{j}^{\prime}, R_{-j}\right)=t_{j}(R) \leq C^{1}\left(R_{-i}, x ; z\right)$,

$$
\begin{aligned}
t_{i}\left(R_{j}^{\prime}, R_{-j}\right)-V_{j}^{\prime}\left(\tau(\mu) ; f_{j}\left(R_{j}^{\prime}, R_{-j}\right)\right) & <V_{i}\left(x ;((\tau(\mu), 0))-C^{1}\left(R_{-i}, x ; z\right)\right. \\
& \leq V_{i}(x ;(\tau(\mu), 0))-t_{j}\left(R_{j}^{\prime}, R_{-j}\right) .
\end{aligned}
$$

Thus, by Lemma B.4, $f\left(R_{j}^{\prime}, R_{-j}\right)$ is not efficient, a contradiction. Thus $x_{i}(R)=x$.Q.E.D.

Proposition B.1: Let $f$ satisfy the four axioms of Theorem 1. Let $R \in\left(\mathcal{R}^{T}(\mathcal{T})\right)^{n}$. Let $z \in Z^{\min }(R)$. Then, for each $i \in N, f_{i}(R) R_{i} z_{i}$.

The proof of Proposition B.1 is similar to that of Proposition A.1. Thus, we omit it.

The rest of the proof of Theorem 2 is similar to that the proof of Theorem 1. Thus, we also omit it.

Q.E.D. 